\title{
Localization of Cortical Phase and Amplitude Dynamics during Visual Working Memory Encoding and Retention
}

\author{
Satu Palva, ${ }^{1}$ Shrikanth Kulashekhar, ${ }^{1,2}$ Matti Hämäläinen, ${ }^{3,4}$ and J. Matias Palva ${ }^{1}$ \\ ${ }^{1}$ Neuroscience Center, University of Helsinki, 00014 Helsinki, Finland, ${ }^{2}$ BioMag Laboratory, HUSLAB, 00029 HUS, Finland, ${ }^{3}$ Athinoula A. Martinos Center \\ for Biomedical Imaging, Massachusetts General Hospital, Charlestown, Massachusetts 02129, and ${ }^{4}$ Harvard-Massachusetts Institute of Technology Division \\ of Health Sciences and Technology, Massachusetts Institute of Technology, Cambridge, Massachusetts 02139
}

Several studies show that the amplitudes of human brain oscillations are modulated during the performance of visual working memory (VWM) tasks in a load-dependent manner. Less is known about the dynamics and identities of the cortical regions in which these modulations take place, and hence their functional significance has remained unclear. We used magnetoencephalography and electroencephalography together with minimum norm estimate-based source modeling to study the dynamics of ongoing brain activity during a parametric VWM task. Early stimulus processing and memory encoding were associated with a memory load-dependent spread of neuronal activity from occipital to temporal, parietal, and frontal cortical regions. During the VWM retention period, the amplitudes of oscillations in theta/alpha- (5-9 Hz), high-alpha- $(10-14 \mathrm{~Hz})$, beta- $(15-30 \mathrm{~Hz})$, gamma- $(30-50 \mathrm{~Hz})$, and high-gamma- $(50-150 \mathrm{~Hz})$ frequency bands were suppressed below baseline levels, and yet, in frontoparietal regions, load dependently strengthened. However, in occipital and occipitotemporal structures, only beta, gamma, and high-gamma amplitudes were robustly strengthened by memory load. Individual behavioral VWM capacity was predicted by both the magnitude of the $\mathrm{N} 1$ evoked response component in early visual regions and by the amplitudes of frontoparietal high-alpha and high-gamma band oscillations. Thus, both early stimulus processing and late retention period activities may influence the behavioral outcome in VWM tasks. These data support the notion that beta- and gammaband oscillations support the maintenance of object representations in VWM whereas alpha-, beta-, and gamma-band oscillations together contribute to attentional and executive processing.

\section{Introduction}

Working memory (WM) maintains and manipulates information (Baddeley, 1996) and is based on the retention of integrated neuronal object representations (Luck and Vogel, 1997; Zhang and Luck, 2008). The binding of sensory feature representations into object representations and the active maintenance of these representations in WM may depend on millisecond-range synchronization of neuronal network oscillations (Fries, 2009; Singer, 2009; Palva et al., 2010b). In human magnetoelectroencophalography (MEG) and electroencephalography (EEG) recordings, the maintenance of visual (Tallon-Baudry et al., 1998; Osipova et al., 2006; Jokisch and Jensen, 2007; Medendorp et al., 2007; Haenschel et al., 2009), auditory (Leiberg et al., 2006; Kaiser et al., 2008) and somatosensory (Haegens et al., 2010) information in WM is associated with increased beta- and gamma-frequency band amplitudes. However, the amplitudes of theta- and alpha-band oscillations also are modulated during WM maintenance (Jensen et al., 2002; Busch and Herrmann, 2003; Onton et al., 2005; Leiberg et al., 2006;

Received 0ct. 25, 2010; revised Jan. 13, 2011; accepted Feb. 2, 2011.

This study was supported by the Academy of Finland, by the Helsinki University Research Funds, the National Institutes of Health National Center for Research Resources Grant P41 RR14075, and National Institute of Biomedical Imaging and Bioengineering Grants 1R01EB009048 and 1R01 EB006385.

Correspondence should be addressed to either Satu Palva or J. Matias Palva, Neuroscience Center, P.0. Box 56, University Helsinki, 00014 Helsinki, Finland. E-mail: satu.palva@helsinki.fi or matias.palva@helsinki.fi.

DOI:10.1523/JNEUROSCI.5592-10.2011

Copyright $\odot 2011$ the authors $\quad 0270-6474 / 11 / 315013-13 \$ 15.00 / 0$
Osipova et al., 2006; Jokisch and Jensen, 2007; Haenschel et al., 2009). Prior studies have used several distinct WM tasks and memory retention intervals, which make it difficult to obtain an integrative view on the insight given by these studies into brain functioning. The difficulties in inferring the physiological roles of scalp-recorded oscillations also stem from the absence of source modeling in most studies. Studies using MEG/EEG (M/EEG) source modeling suggest that the amplitude modulations in visual WM (VWM) tasks arise in occipital and parietal brain regions (Osipova et al., 2006; Jokisch and Jensen, 2007; Medendorp et al., 2007). Functional magnetic resonance imaging (fMRI) has, in addition, revealed VWM maintenance-related activity in several frontal and temporal regions as well as in the cingulate and insular cortices (Prabhakaran et al., 2000; Rowe et al., 2000; Munk et al., 2002; Pessoa et al., 2002; Sakai et al., 2002; Linden et al., 2003; Todd and Marois, 2004; Mohr et al., 2006; Axmacher et al., 2007). Intracranial recordings of epileptic patients also show amplitude modulations during VWM maintenance in widespread occipital, temporal, parietal, and frontal cortical regions (Raghavachari et al., 2001; Howard et al., 2003; Mainy et al., 2007; Sederberg et al., 2007; Meltzer et al., 2008), and in the hippocampus (Axmacher et al., 2007). Indeed, by using fMRI priors in source modeling, sustained event-related activity (Wibral et al., 2008; Scheeringa et al., 2009; Robitaille et al., 2010) as well as theta- (Morgan et al., 2010) and alpha-band (Grimault et al., 2009) amplitude modulations have been identified in occipital, temporal, and frontal cortical regions. 
In the present study, we set out to characterize comprehensively the neuronal substrates underlying a parametric and psychophysically well validated VWM task using concurrently acquired M/EEG data. We used data-driven statistical source space analyses to map in individual cortical anatomy the dynamics of early stimulus processing and later amplitude modulations of ongoing oscillations. The investigation aimed at identifying the cortical regions and time-frequency windows where brain activity is modulated by VWM task performance, correlated with memory load, and/or predictive of individual behavioral performance.

\section{Materials and Methods}

Task and stimuli. We used a delayed matching-to-sample task where the subjects' were presented a sample stimulus that contained one to six squares, and had to be sustained in WM and then compared with a test stimulus (Luck and Vogel, 1997; Palva et al., 2010a,b) (supplemental Fig. $1 \mathrm{~A}$, available at www.jneurosci.org as supplemental material). The sample stimulus was presented for $0.1 \mathrm{~s}$ and was followed by a $1 \mathrm{~s}$ memory retention period, after which a test stimulus was presented for $0.5 \mathrm{~s}$. The subjects indicated with a forced-choice left- or right-hand thumb twitch whether the stimuli were "same" or "different." The hand response correspondence was randomized across subjects. After this primary response, the subjects indicated whether or not they felt sure about their response with a second go/no-go, respectively, twitch of the same thumb.

Behavioral performance. Accuracy was assessed as the proportion of correct responses from among all responses (supplemental Fig. $1 \mathrm{~B}$, available at www.jneurosci.org as supplemental material). Reaction time was given by the time from the sample stimulus onset to the onset of the electromyogram (see Subjects and recordings, below) activity corresponding to the primary response (supplemental Fig. $1 C$, available at www.jneurosci.org as supplemental material). All analyses were based on artifact-free trials with correct behavioral responses.

Subjects and recordings. Thirteen healthy right-handed (age, $28 \pm 3$ years, mean $\pm \mathrm{SD} ; 4$ females) volunteers with normal or corrected-tonormal vision participated in this study. The 366-channel M/EEG data were recorded with 204 planar gradiometers, 102 magnetometers, and 60 EEG electrodes (Elekta Neuromag) at a $600 \mathrm{~Hz}$ sampling rate throughout the experiment (supplemental Fig. $1 D$, available at www.jneurosci.org as supplemental material). The thumb-twitch responses were recorded with electromyography (EMG) of abductor/flexor pollicis brevis and detected with an automatic algorithm. All EMG responses were also verified visually. The electro-oculogram was used to detect ocular artifacts. Trials with the electro-oculogram signal exceeding $50 \mu \mathrm{V}$ were excluded from further analysis. The MaxFilter software (Elekta Neuromag) was used to suppress extracranial noise and to colocalize the signal space data from different recording sessions and subjects. For cortical surface reconstructions, we recorded T1-weighted (magnetization-prepared rapid-acquisition gradient echo) anatomical MR images at a $\leq 1 \times 1 \times 1 \mathrm{~mm}$ resolution with a 1.5 T MRI scanner (Siemens) (supplemental Fig. 1E, available at www. jneurosci.org as supplemental material). This study was approved by the ethical committee of Helsinki University Central hospital and was performed according to the Declaration of Helsinki. Written informed consent was obtained from each subject before the experiment.

Data analysis, Morlet wavelet transform. Each channel, $y_{j}(t)$, of the single-trial M/EEG time-series data, $Y(t)$, with $n_{\mathrm{c}}$ channels $j=1 \ldots$ $n_{c}$, was filtered into 36 frequency bands, $f, f=3 \ldots 90 \mathrm{~Hz}$, with a bank of Morlet wavelets, $h(t, f)$, so that the complex filtered signal $y_{\mathrm{F}}(t, f)$ is given by $y_{\mathrm{F}}(t, f)=y(t) * h(t, f)$, where ${ }^{*}$ denotes convolution and $h(t, f)=A \exp \left(-t^{2} / 2 \sigma_{t}^{2}\right) \exp (2 i \pi f t)$ (supplemental Fig. 1G, available at www.jneurosci.org as supplemental material). The timedomain SD of the wavelet is given by $\sigma_{t}=m / 2 \pi f$, where we selected $m=5$ to define a compromise between time and frequency resolution, and $f$ is the center frequency of the wavelet.

Finite impulse response filtering. In the second stage of this study, we used a set of paired high- and low-pass finite impulse response (FIR) filters that gave a better temporal resolution than the Morlet wavelets and represented the spectral content captured by four adjacent wavelets with a single filter in each of the four frequency bands of interest delineated by the prior wavelet analysis. The stop and pass bands of the high-pass filters, and the pass and stop bands of the low-pass filters, respectively, were as follows (in $\mathrm{Hz}$ ): theta/alpha $[2.375,4.75,6.65,9.5]$; high-alpha $[4.75,9.5,13.3,19.0]$; beta $[9.5,19.0,26.6,38.0]$; gamma $[14.25,28.5$, $37.05,49.875]$; and high-gamma $[48.75,75.0,112.5,150.0]$. The parameters for the FIR filters used in evoked response (ER) analyses were (in $\mathrm{Hz})[0.01,0.1,25,45]$ and $[1,2,25,45]$.

Forward and inverse modeling. The FreeSurfer-anatomical-MRI analysis software (http://surfer.nmr.mgh.harvard.edu/) was used for automatic volumetric segmentation of the MRI data, surface reconstruction, flattening, cortical parcellation, and labeling with the Freesurfer/ Destrieux atlas (Dale et al., 1999; Fischl et al., 1999; Fischl et al., 2001, 2002, 2004; Ségonne et al., 2004; Desikan et al., 2006) (supplemental Fig. $1 H$, available at www.jneurosci.org as supplemental material). The MNE software (http://www.nmr.mgh.harvard.edu/martinos/userInfo/data/ sofMNE.php) was used for creating three-layer boundary element conductivity models, cortically constrained source models, for the M/EEGMRI colocalization, and for the preparation of the forward and inverse operators (Hämäläinen and Sarvas, 1989; Hämäläinen and Ilmoniemi, 1994; Nenonen et al., 1994; Mosher et al., 1999; Lin et al., 2006) (supplemental Fig. $1 I, J$, available at www.jneurosci.org as supplemental material). The source models for each individual were based on tessellated and decimated cortical surfaces where the dipoles were fixed normal to the surface and had an $\sim 7 \mathrm{~mm}$ source-to-source separation. Depending on the brain size, the source models had a total of $n_{d}=6000-8500$ sources in the two cerebral hemispheres.

M/EEG sensor signals, $Y$, are linearly related to current strengths in $n_{d}$ source dipoles, $X=\left[x_{k}\right], k=1 \ldots n_{d}$, so that $Y(t)=\Gamma X(t)+N(t)$, where $\Gamma$ is the lead field matrix (i.e., the forward operator), and $N$ denotes noise. We obtained $X(t)$ from measured $Y(t)$ by using a minimum-norm estimator (Hämäläinen and Sarvas, 1989; Nenonen et al., 1994), so that $X(t)=$ $\mathrm{M} Y(t)=C_{s} \Gamma^{\mathrm{T}}\left(\Gamma C_{s} \Gamma^{\mathrm{T}}+\lambda^{2} C_{n}\right)^{-1} Y(t)$, where $\mathrm{M}$ is the inverse operator, $\lambda^{2}$ is a regularization parameter, $C_{s}$ is the source covariance matrix, and $C_{n}$ is the noise covariance matrix. We used $\lambda^{2}=0.05$ and a multiple of the identity matrix as $R$, scaled so that the norm of $\Gamma C_{s} \Gamma^{\mathrm{T}}$, whitened spatially using $C_{n}$, equals the number of channels (see MNE manual). The inverse operator, M, was prepared separately for each wavelet filter or FIR filter frequency with the noise covariance matrices obtained from filtered single-trial prestimulus baseline windows of all trials (supplemental Fig. $1 K$, available at www.jneurosci.org as supplemental material). The complex inverse solution, $X_{\mathrm{F}}=\left[\mathrm{x}_{\mathrm{F}, k}\right]$, was obtained from the inverse estimates of the real, $Y_{\mathrm{F}, \mathrm{RE}}$, and imaginary, $Y_{\mathrm{F}, \mathrm{IM}}$, parts of the filtered data $Y_{\mathrm{F}}$, so that $X_{\mathrm{F}}=\mathrm{M}_{\mathrm{F}} Y_{\mathrm{F}, \mathrm{RE}}(t)+i \mathrm{M}_{\mathrm{F}} Y_{\mathrm{F}, \mathrm{IM}}(t)$, where $i$ is the imaginary unit (supplemental Fig. $1 L$, available at www.jneurosci.org as supplemental material).

Surface parcellations. We used two cortical parcellation strategies in this study. The aims of source-model parcellation were to compress single-trial data and to transform the data into nameable anatomical regions that were common to all subjects. A parcellation denotes the grouping of vertices into a number of patches. In the Morlet waveletbased analyses, we employed the 156 labels of the FreeSurfer atlas (Fischl et al., 2002) covering the entire cortical surface (see supplemental Fig. $1 H$, available at www.jneurosci.org as supplemental material) and then further split the largest patches to obtain the anatomical parcellation, $P_{\text {AN240 }}$, comprising a total of 240 patches (supplemental Fig. $1 M$, available at www.jneurosci.org as supplemental material). The splitting was carried out iteratively so that in each iteration the patch having the greatest mean number of vertices was selected and split along the one of the three axes (anterior-posterior, lateral-medial, ventral-dorsal) that had the largest mean variance of vertex locations. This procedure gives an anatomical parcellation wherein the cut directions are identical for every subject. The individual anatomical data were thus directly comparable in group statistics of the subject population without a need for morphing the data across subjects (Palva et al., 2010b). The FIR filter-based analyses were performed with two parcellations: the single-trial data were compressed to and averaged with an individual cluster parcellation of 700 clusters, $P_{C L 700}$; group statistics were then performed with an anatomical parcellation with 700 patches, $P_{\text {AN700 }}$, after morphing the data from each 
A
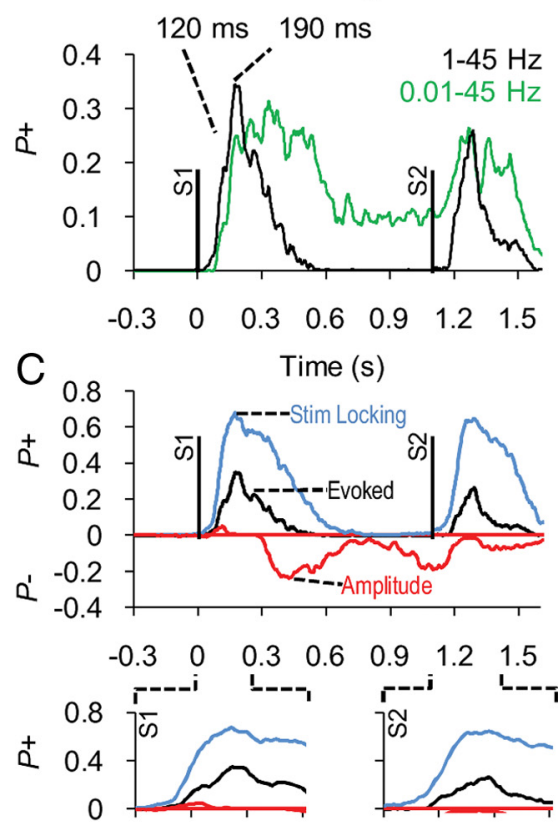

$\begin{array}{llll}0 & 0.1 & 0.2 & 0.3\end{array}$

Time (s)

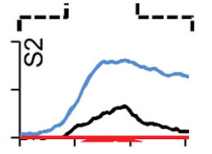

$\begin{array}{llll}1.1 & 1.2 & 1.3 & 1.4\end{array}$

Time (s)
B

Load

+

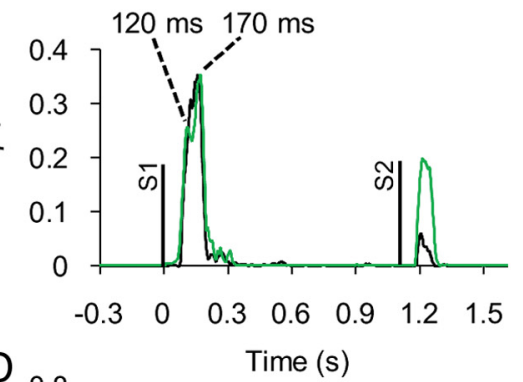

$\mathrm{D}$

$+0.6$

$a^{\prime}$

0
-0.2
-0.4

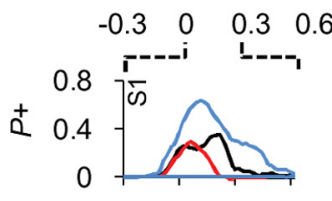

$\begin{array}{llll}0 & 0.1 & 0.2 & 0.3\end{array}$

Time (s) phases of a subset of vertices were shifted by $\pi$. This was done because in a source model with fixed dipole orientations, the time series for a single dipole in one wall will be $180^{\circ}$ out of phase with the time series of the dipole in the opposite wall. These to-be-shifted vertices were identified from the within-patch phase synchrony estimates as that minority of vertices that had a phase difference of approximately $\pi$ with the majority of vertices having a zero-lag phase difference. Vertices with an opposite polarity arise in cortical patches where the patch, for instance, contains both sides of the same sulcus. The phase shifting prevents vertices with opposite polarities from canceling each other when the vertex time series are averaged into the patch time series.

Evoked response, amplitude, and stimuluslocking analyses. We used the collapsed inverse estimates, $\mathrm{X}_{\mathrm{F}, \mathrm{P}, r}(t, f)$, of single trials $r, r=1 \ldots$ $n_{s}$, for cortex-wide mapping of evoked responses and the phase locking of ongoing activity to the sample stimuli [hereafter called "stimulus locking" (SL)] as well as for the mapping of event-related amplitude dynamics (supplemental Fig. 1O, available at www. jneurosci.org as supplemental material). Stimulus locking was quantified with the phaselocking value, $P L V(t, f)$ (also known as the phase-locking factor), that was given for each patch $a=1 \ldots n_{p}$ by $P L V=n_{s}^{-1} \mid \Sigma_{r}\left(\mathrm{x}_{\mathrm{F}, \mathrm{P}, a}\right)$ $\left.\left|\mathrm{x}_{\mathrm{F}, \mathrm{P}, a}\right|\right) \mid$ (Sinkkonen et al., 1995). The averaged event-related amplitude envelopes, $A(t, f)$, were given by $A=n_{s}^{-1} \Sigma_{\mathrm{r}}\left(\left|\mathrm{x}_{\mathrm{F}, \mathrm{P}, a}\right|\right)$ (TallonBaudry et al., 1996; Palva et al., 2005) and the evoked responses, $E R(t, f)$, simply by the averaging the real parts of the time series so that $E R=n_{s}^{-1} \Sigma_{\mathrm{r}}\left[\operatorname{Re}\left(x_{\mathrm{F}, \mathrm{P}, a}\right)\right]$,

Group statistics: primary statistical analyses. Before the statistical group analyses (Figs. 1, 2; see Figs. 4, 5, 7; supplemental Figs. $1 P, 2-8$, individual's $P_{C L 700}$ to that individual's $P_{A N 700}$ (Palva et al., 2010b). The individual subject's parcellations were obtained by (1) inverse modeling baseline data that was FIR filtered into a frequency band from 150 to 224 $\mathrm{Hz}$ (filter parameters as above: $[96.85,149.0,223.5,298.0]$ ), (2) evaluating vertex-by-vertex phase-locking, value-based, 1:1 phase-synchrony matrices within each of the 150 patches of the Destrieux atlas (3) performing hierarchical clustering of source space vertices so that always the pair of vertices (or vertex clusters) that had the greatest phase-locking value were clustered. The hierarchical clustering was continued until 700 clusters remained. The hierarchical clustering approach was thus similar to the one described earlier (Palva et al., 2010b), but here the clusters were limited to the 150 anatomical patches of the Destrieux atlas. This clustering compressed single-trial source space data with minimal loss of information because the clusters reflected vertices that were highly correlated in the original recording and/or in the inverse transform. The $150-224 \mathrm{~Hz}$ band was used for this purpose because the amplitude [and signal-to-noise ratio (SNR)] of neuronal activity therein is negligible and hence the phase-synchrony estimates describe very well the correlations inherently present in the M/EEG inverse solutions.

The time series of the $\sim 7000$ source vertices were collapsed into time series of the cortical patches (supplemental Fig. $1 \mathrm{~N}$, available at www. jneurosci.org as supplemental material) so that the collapsed inverse solution, $X_{\mathrm{F}, \mathrm{P}}=\left[x_{\mathrm{F}, \mathrm{P}, l}\right], l=1 \ldots n_{p}$, is given by $X_{\mathrm{F}, \mathrm{P}}=\Pi\left(X_{\mathrm{F}}, P\right)$, where $\Pi$ is a collapse operator, $P$ is the parcellation, $P=\left[p_{l}\right]$, and $n_{p}$ is the number of patches, $p_{l}$, in $P$. In the Morlet wavelet analyses, we defined $\Pi$ so that the patch time series was obtained from vertex time series simply by averaging over the time series of the individual vertices of within that patch. In the FIR filter-based analyses, $\Pi$ was defined so that the amplitudes of the individual vertex time series were averaged as above but the available at www.jneurosci.org as supplemental material), the individual subject's $P L V(t, f), A(t, f)$, and absolute-value evoked responses $(E R)$ were baseline corrected by subtracting the mean of the pre-sample-stimulus period from -450 to $-150 \mathrm{~ms}$ (Morlet wavelet data) or from -700 to $-100 \mathrm{~ms}$ (FIR filter data). The data were then pooled across subjects by applying a statistical test to obtain $P L V_{\mathrm{S}}$ and $A_{\mathrm{S}}$ for each time frequency element of PLV and $A$, and separately for each VWM load, $L_{\mathrm{M}}=1,2,3$, 4,5 , and 6 . In the average condition and for all VWM loads separately, the baseline-corrected $P L V, A$, and $E R$ were tested against a null hypothesis of $P L V=0, A=0$, and $E R=0$ by using the Wilcoxon signed rank or Student's $t$ test. These conditions thus address whether the studied values are significantly different from the mean baseline value. The correlations of the PLV, A, and ER values in each time, frequency, and cortical location with memory load (load condition) were estimated by using the Spearman's rank correlation test across the memory loads $L_{\mathrm{M}}=1,2,3,4$, 5 , and 6 so that the values were first rank transformed within each subject and then used in the statistical group analysis across subjects. Individual variability in response magnitudes thus did not influence the outcome of the analysis.

Post hoc analyses. Post hoc analyses were performed to a selection of brain regions showing significant modulation by the VWM load (Fig. 3; see Fig. 6; supplemental Figs. 9, 10, available at www.jneurosci.org as supplemental material). The peak latencies and amplitudes were estimated from the peaks searched from the signal in two time windows (80-120 and 160-200 ms, respectively, for the first and second peak). The mean ER magnitudes were estimated from the same time windows, whereas the mean oscillation amplitudes were estimated from the late 
retention period time window (700-1000 ms). The selection of time window did not affect the results qualitatively.

The correlations of the ER and oscillation amplitude with the individual memory capacity were identified so that we first obtained mean $E R$ and $A$ values separately for each of the six memory loads from 1 to 6 , fitted a thirdorder polynomial to this mean-as-a-functionof-load series, and subtracted the linear trend. The "predicted capacity values" were then obtained by finding the first positive peak. The correlation between the predicted and real capacity values was estimated by the Spearman rank correlation test.

\section{Results}

Behavioral performance

During the M/EEG recordings, the subjects performed a delayed match-to-sample VWM task (supplemental Fig. $1 A$, available at www.jneurosci.org as supplemental material) (Luck and Vogel, 1997; Palva et al., 2010a,b) wherein they memorized a $0.1 \mathrm{~s}$ sample stimulus containing one to six colored squares. A test stimulus appeared $1 \mathrm{~s}$ after the offset of the sample, and in $50 \%$ of the trials one square in the test had a different color than that square in the sample. The subjects indicated with a forced-choice leftor right-hand thumb twitch whether or not the sample was identical to the test. As reported for earlier (Luck and Vogel, 1997) and present (Palva et al., 2010b) data, the behavioral accuracy decreases with increasing memory load (supplemental Fig. 1B, available at www.jneurosci.org as supplemental material), while the reaction times become longer (supplemental Fig. $1 C$, available at www.jneurosci.org as supplemental material). Thus, this VWM task yields quantitatively similar psychophysical data during repeated $\mathrm{M} / \mathrm{EEG}$ recordings as obtained outside the neuroimaging context.

\section{Stimulus-processing and memory-encoding period}

The aim of this study was to identify the cortical sources and dynamics of VWMrelated neuronal activities and to address their functional significance in VWM by finding the components that are correlated with memory load and/or predict individual psychophysical performance. The processing of the sample and test stimuli was examined first by evaluating absolute-value ERs with two broad-band FIR bandpass filters (1-45 and $0.01-45 \mathrm{~Hz}$ ) across the six memory load conditions. The data were summarized into waveforms indicating the fraction of brain regions where the ERs were significantly $[p<0.001$, false discovery rate (FDR) corrected] above the baseline level. Both sample and test stimuli were associated with ERs lasting approximately the first $400 \mathrm{~ms}$ from stimulus onset (Fig. 1A). Interestingly, the ERs obtained with the lower high-pass filter SPG, superior parietal gyrus.
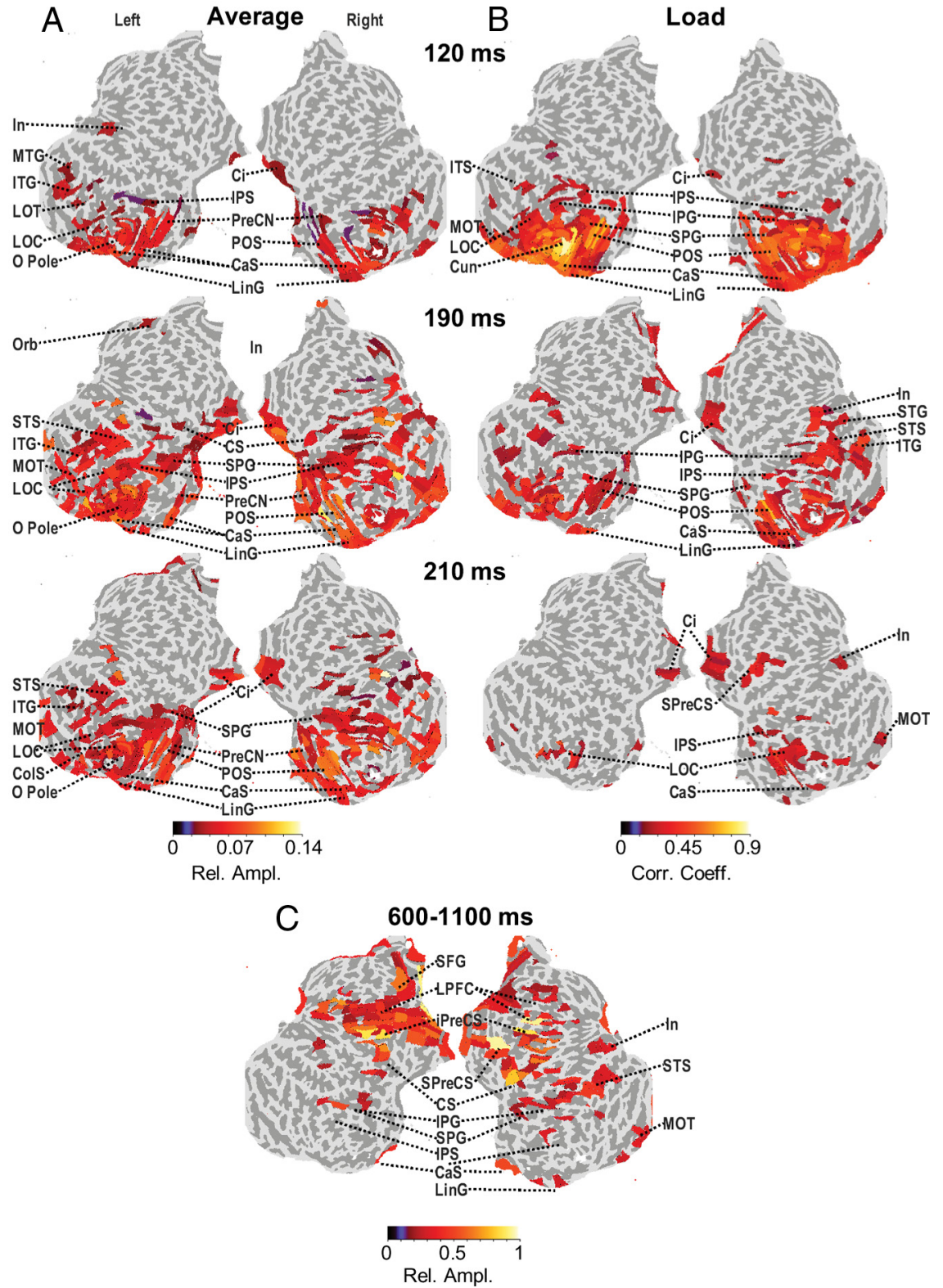

Figure 2. Evoked responses spread from visual cortex to frontal, cingulate, and insular cortices. $\boldsymbol{A}$, The temporal evolution of the evoked response in the 1-45 Hz band is visualized at latencies of 120 (P1), 190 (N1), and $210 \mathrm{~ms}$ after the sample stimulus in the average condition (for a more complete time series, see supplemental Figs. 2, 3, available at www.jneurosci.org as supplemental material). The anatomical map shows the complete flattened left and right cortical surfaces. Color values indicate the strength of the evoked response in those cortical regions where it was significant ( $p<0.001$, multiple comparisons corrected) at the given latency. $\boldsymbol{B}$, The topographies of evoked response components that were significantly correlated with memory load. $\boldsymbol{C}$, The topography of the slow retention period component revealed by the $0.01-45 \mathrm{~Hz}$ filter (see Fig. $1 \mathrm{~A}$ ) in the average condition. CaS, Calcarine sulcus; $\mathrm{CS}$, central sulcus; Ci, cingulate; ColS, collateral sulcus; iPreCS, inferior precentral sulcus; In, insula; LinG, lingual gyrus; LOC, lateral occipital cortex; MOT, medial occipitotemporal cortex; Orb, orbital; POS, parieto-occipital sulcus; PreCN, precuneus; $\mathrm{SFG}$, superior frontal gyrus; $S$ PreCS, superior precentral sulcus; STS, superior temporal sulcus; Rel. Ampl., relative amplitude; Corr. Coeff., correlation coefficient; Cun, cuneus; IPG, inferior parietal gyrus; MTG, middle temporal gyrus; OPole, occipital pole;

$(0.01-45 \mathrm{~Hz})$ remained above the baseline throughout the retention period and thus contained a slow baseline shift-like component. We then estimated the effect of memory load on the evoked responses by using Spearman's rank correlation test $(p<0.001$, FDR corrected), which revealed that the ER components from 60 to $200 \mathrm{~ms}$, but not those after $200 \mathrm{~ms}$ from stimulus onset, were strongly modulated by the number of objects in sample stimuli (Fig. $1 B$ ). One should note that in the present experiment the 
A
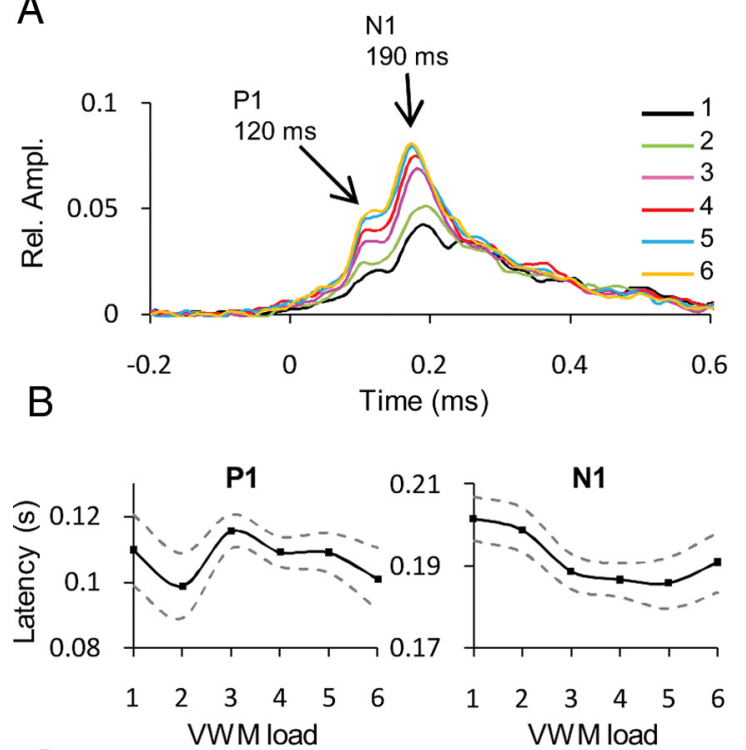

C

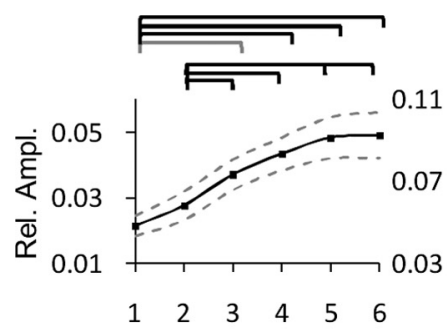

D VWMload

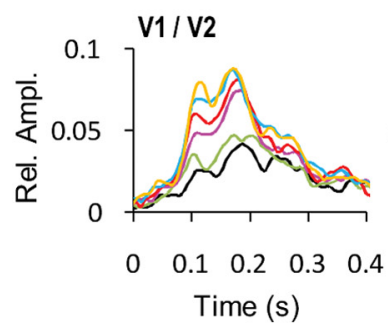

E

P1

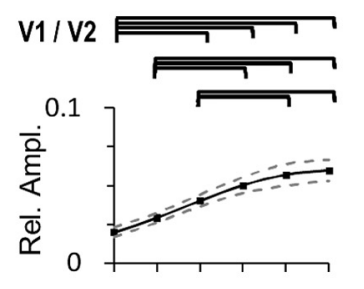

$\begin{array}{llllll}1 & 2 & 3 & 4 & 5 & 6\end{array}$

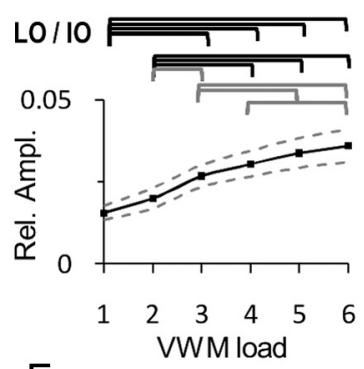

F

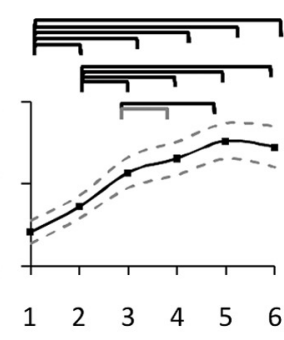

VWM load

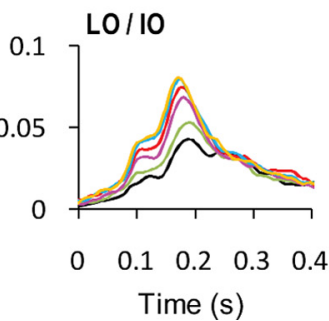

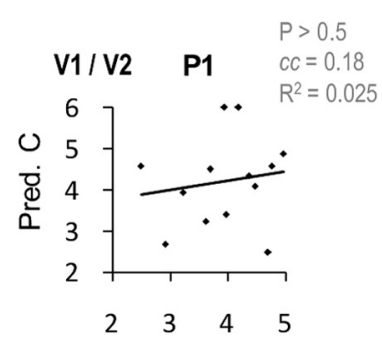

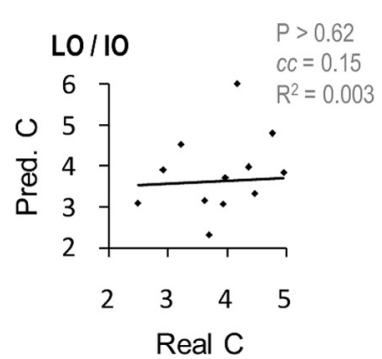

N1
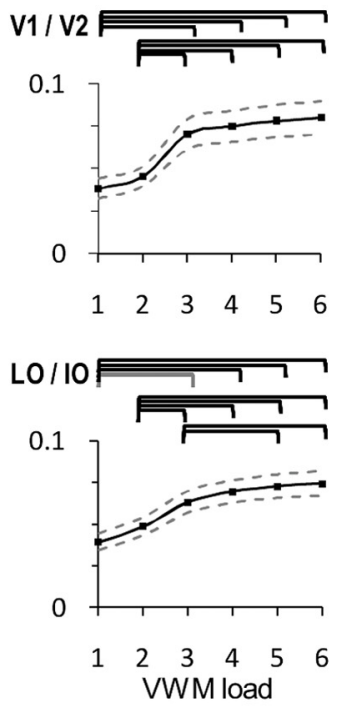

Figure 3. The $\mathrm{N} 1$ evoked response component is correlated with individual memory capacity. $\boldsymbol{A}, \mathrm{The} 1-45 \mathrm{~Hz}$ band evoked responses obtained separately for each memory load (colors as in the figure) were averaged across those cortical regions that were significantly different from the baseline period between 50 and 250 ms from the sample stimulus onset. $\boldsymbol{B}$, The memory load sensitivity of the P1 and N1 component peak latencies (black lines; dashed lines indicate SEMs across the latency values of individual subjects). $\boldsymbol{C}$, The memory load sensitivity of P1 and N1 component amplitudes averaged across time windows of $80-120$ and $180-220$ ms, respectively (black line; dashed lines indicate SEMs across subjects). The brackets indicate the memory load pairs between which the difference was significant in a paired $t$ test across subjects [black lines: $p<0.01$; gray lines: $p<0.05$; both are Bonferroni corrected with the number of tests (15)]. These post hoc statistics corroborate the observations in Figures $1 B$ and $2 B$. D. Evoked responses (see $\boldsymbol{A}$ ) averaged separately across V1/V2 and over L0/I0 cortical regions show that the P1 component is most pronounced in V1/V2. $\boldsymbol{E}$, Mean peak amplitudes and SEMs in V1/V2 and over LO/I0 averaged (see $\boldsymbol{C}$. While P1 was almost linearly dependent on memory load, likely because of the increasing stimulus intensity, N1 showed a clear plateau at high memory loads. $\boldsymbol{F}$, In V1/V2, the plateau onset of the individual subjects' N1 component magnitudes (Pred. C in $\boldsymbol{E}$ ) predicted the individual behavioral memory capacity (Real C). Rel. Ampl., Relative amplitude.

number of objects covaried with the overall complexity of the stimulus and hence the strengthening of ERs with increasing memory load may be at least partly attributed to a concurrent change in physical stimulus properties.

ERs are generally thought to be generated by additive evoked activity but may also be influenced by event-related amplitude changes of non-zero mean oscillations (Nikulin et al., 2007; de Munck and Bijma, 2010) and phase resetting (Makeig et al., 2002) of ongoing activity. To consider the contributions of these mechanisms into the generation of the ERs in the present VWM task, we quantified the phase locking of ongoing activity to the sample and test stimulus onsets (i.e., SL) and evaluated the peristimulus amplitude dynamics by using the same broad-band filters that were used for ERs (Palva et al., 2005). The dynamics of SL was similar to that of ERs, but SL was significant in a greater fraction of cortical regions (Fig. 1C). The amplitude of ongoing cortical broad-band activity, on the contrary, was only briefly strengthened above the baseline level at around $100 \mathrm{~ms}$ after the sample stimulus onset and thereafter suppressed below the baseline level for the rest of trial. Like ERs, both SL and the early amplitude enhancement were robustly correlated with memory load (Fig. 1D).

We next identified the cortical regions involved in the processing of sample stimuli. The first significant ER components 
( $p<0.001$, FDR corrected) were found at around $70 \mathrm{~ms}$. As the broad-band filter provided a good temporal resolution, we identified the ER sources in 10-20 ms steps (supplemental Figs. 2, 3, available at www.jneurosci.org as supplemental material). The evoked activity spread rapidly from V1/V2 to higher level extrastriate, occipitotemporal, and parietal regions. One should note here, however, that the inverse modeling method used here (MNE) provides a distributed source estimate that is likely to be more widespread than the true cortical source. Hence, the source reconstructions contain cross talk between neighboring cortical patches in spatial scales of a few centimeters (Hauk et al., 2011). The $\mathrm{P} 1$ peak at $120 \mathrm{~ms}$ was observed in distributed visual areas in the occipital and inferior temporal cortices including the lateral occipitotemporal cortex (LOT), inferotemporal gyrus (ITG), and the intraparietal sulcus (IPS), as well as in the insular and cingulate cortices (Fig. 2A). The N1 peak at $190 \mathrm{~ms}$ was observed also in distributed regions in the posterior parietal cortex (PPC) as well as in central and superior temporal sulci. This constellation of occipital, temporal, and parietal regions was sustained for approximately $50 \mathrm{~ms}$, after which it gradually degraded. Broadband SL was strongest in the same network of brain regions as the evoked response, but with a more smeared temporal evolution. This was expected because the phase estimates of ongoing activity, by definition, incorporate information across a time window that is dependent on the characteristic time scales of the activity itself. This may, in part, also explain the greater sensitivity of SL compared with ERs. It is interesting to also note that SL revealed early stimulus processing in several frontal cortical regions including the frontal eye fields (FEFs) and lateral prefrontal cortex (LPFC) as well as in anterior insula (supplemental Figs. 2, 3, available at www.jneurosci.org as supplemental material). In contrast, the brief broad-band amplitude enhancement was limited to striate cortex and extrastriate regions surrounding the occipital pole (supplemental Figs. 2, 3, available at www. jneurosci.org as supplemental material).

VWM load strengthened ERs, amplitudes, and SL mostly in the same regions that were identified in the average condition (Fig. 2 B; supplemental Figs. 4, 5, available at www.jneurosci.org as supplemental material). While the memory load effect at P1 peak was limited to occipital, occipitotemporal, and posterior parietal cortices, the VWM load strengthened N1 peak additionally in superior temporal, superior and orbitofrontal, insular, and cingulate cortices. The correlation of amplitude with memory load was robust throughout the posterior cortical networks and well colocalized with the spatiotemporal evolution of ERs (supplemental Figs. 4, 5, available at www.jneurosci.org as supplemental material). The SL, in contrast, was also strongly correlated with memory load in several more anterior structures, including the anterior cingulate and insula as well as paracentral and superior precentral sulci (supplementary motor area and FEF, respectively). Hence, these results suggest that in these data in posterior brain regions, ERs could have been generated by additive stimulus-evoked activity, but in the anterior brain regions phase resetting of ongoing oscillations contributed to the generation of ERs.

Finally, we also localized the sustained slow evoked component that was salient in the ER obtained with $0.01-45 \mathrm{~Hz}$ filter (Fig. 1A). After the termination of the transient ER and in the time window from 600 to $1100 \mathrm{~ms}$, the slow component was strongest in the LPFC and cingulate cortices as well as in paracentral, precentral, and postcentral areas (Fig. 2C). More posteriorly, the slow component was also observed in PPC and lingual gyrus.
Moreover, while the slow retention period ER component was largely colocalized with frontal load-dependent oscillation amplitude modulations (see Cortical sources of retention period modulations of oscillation amplitudes, below), it was uncorrelated with memory load, which suggests that this component might not be related to the amplitude changes of alpha or faster oscillations through the non-zero mean mechanism. The insensitivity to VWM load of the slow ER component suggests that it could reflect arousal- or alertness-related fluctuations (Monto et al., 2008; Schroeder and Lakatos, 2009).

\section{Early evoked responses are correlated with VWM performance}

To shed light on the functional significance of neuronal activity reflected in the ERs, we characterized the early components in more detail with post hoc analyses separately for each memory load condition in those cortical regions wherein the ERs were significantly correlated with memory load (Fig. $2 B$ ). We first averaged the absolute-value ER time series across this selection (Fig. $3 A$ ) and then used peak detection to estimate the mean latencies and amplitudes of the P1 and N1 peaks (at $\sim 120$ and $190 \mathrm{~ms}$, respectively) across the subject population (Fig. 3B). The peak latencies were relatively stable across the memory loads, and while N1 appeared to be earlier for higher VWM loads, this tendency was not significant. The peak amplitudes were enhanced with VWM load (Fig. 3C). We then focused the analysis into two anatomically defined regions of interest: one approximately containing V1 and V2 (calcarine fissure, cuneus, occipital pole, and lingual gyrus), and another comprising the remaining extrastiate visual regions in lateral occipital (LO) and inferior occipital (IO) cortices (Figs. $2 \mathrm{~B}, 3 \mathrm{D}$ ). The strength of $\mathrm{P} 1$ was larger in the $\mathrm{V} 1 / \mathrm{V} 2$ than in the LO/IO selection (Fig. 3D), but in both regions of interest the strength of P1 and N1 was increased with the VWM load (Fig. 3E). Interestingly, while the amplitude of the P1 was essentially linearly dependent on the number of objects in the sample stimulus, the amplitude of N1 increased from one to three objects and plateaued thereafter. This plateau onset is near the mean behavioral memory capacity in these data $(4.1 \pm 0.2$, mean \pm SEM) (Palva et al., 2010b), and, hence, we investigated whether this plateau onset in individual subjects' ERs would be correlated with their VWM capacity. We estimated the plateau onset by detecting the first positive peak in the difference of thirdand second-order polynomials that were least squares fitted to the series of ER peak amplitudes for the six memory loads (see Materials and Methods). The correlation of this ER-predicted capacity value with the real behavioral capacity value was then evaluated with Spearman's correlation test. Intriguingly, this analysis showed that strength of N1 was, indeed, positively correlated with memory capacity in V1/V2 but not in LO/IO (Fig. $3 F$ ). $\mathrm{P} 1$ was uncorrelated with behavioral capacity in both $\mathrm{V} 1 / \mathrm{V} 2$ and $\mathrm{LO} / \mathrm{IO}$.

Because early ER components are well known to reflect such physical stimulus properties, the memory load dependence of P1 in these data cannot be used to infer a role for in VWM encoding. However, the correlation of $\mathrm{N} 1$ with individual behavioral memory capacity indicates that ERs in this latency range already reflect VWM-related processing and are not fully determined by the physical properties of the stimuli.

\section{Oscillation dynamics in VWM}

The principal aim of this study was to identify the cortical regions where non-stimulus locked ongoing oscillations are correlated with the VWM retention, memory load, or behavioral perfor- 

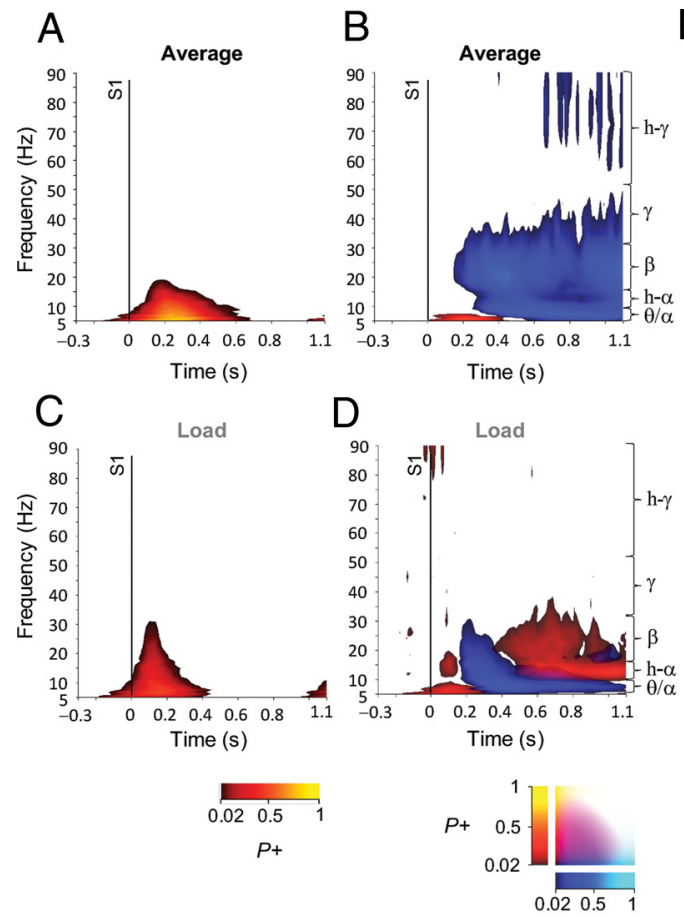

P-

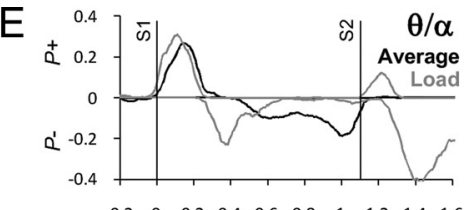

$\begin{array}{llllllllll}-0.2 & 0 & 0.2 & 0.4 & 0.6 & 0.8 & 1 & 1.2 & 1.4 & 1.6\end{array}$

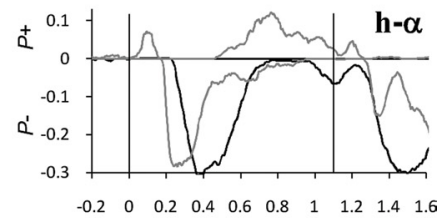

$+$

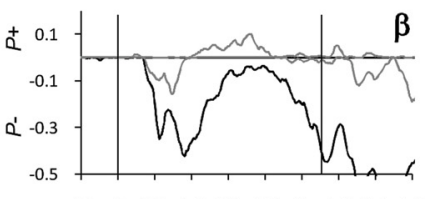

$\begin{array}{lllllllllll}-0.2 & 0 & 0.2 & 0.4 & 0.6 & 0.8 & 1 & 1.2 & 1.4 & 1.6\end{array}$

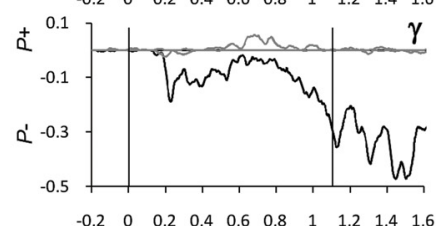

$\begin{array}{llllllllllllll}0.2 & 0 & 0.2 & 0.4 & 0.6 & 0.8 & 1 & 1.2 & 1.4 & 1.6\end{array}$

$$
+0.04
$$

0.02

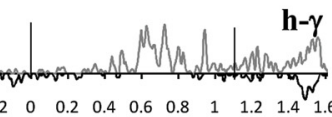

Time (s)

Figure 4. Phase and amplitude dynamics of VWM-related oscillations. A, TFR of stimulus locking in the average condition. Time, relative to the sample stimulus, is displayed on the $x$-axis, and the wavelet frequency is displayed on the $y$-axis. The fraction of cortical regions which showed significant $\left(P^{+}, p<0.001\right.$, multiple comparisons corrected) stimulus locking is shown on the color scale. $\boldsymbol{B}$, TFR of the fraction of cortical regions showing significant positive $\left(P^{+}\right)$and/or negative $\left(P^{-}\right)$changes in amplitude from the baseline level in the average condition (scale as in $\boldsymbol{A}$ ). C, TFR of stimulus locking in the load condition showing the fraction of cortical regions that were significantly positively $\left(P^{+}\right)$and/or negatively $\left(P^{-}\right)$correlated with memory load. $\boldsymbol{D}$, TFR of amplitude dynamics in the load condition (scale as in $\boldsymbol{C}$. $\boldsymbol{E}$, Peristimulus amplitude changes in the average and load conditions were also evaluated with bandpass filters broader than the Morlet wavelets (see Materials and Methods) separately for each of the characteristic frequency bands. Time is displayed on the $x$-axis, and the fraction of cortical regions showing significant amplitude changes from the baseline (black lines, $p<0.001$, corrected) or significant correlations with memory load (gray lines, $p<0.001$, corrected) on the $y$-axis. $\theta / \alpha$, Theta/alpha; $\mathrm{h}-\alpha$, high alpha; $\beta$, beta; $\gamma$, gamma; $\mathrm{h}-\gamma$, high gamma.

mance. ERs, reflecting phasic stimulus-locked brain activity, lasted until $\sim 400 \mathrm{~ms}$ from the sample stimulus onset and were VWM capacity sensitive already at $\sim 200 \mathrm{~ms}$, suggesting that memory encoding could be completed before $400 \mathrm{~ms}$. We thus consider the VWM retention period in these data to be from 400 to $1100 \mathrm{~ms}$ from the sample stimulus onset. The majority of studies on the role of oscillations in VWM have used retention periods of up to $3 \mathrm{~s}$. It has, however, been suggested that the mechanism of memory maintenance may change after the first second of memory retention (Todd and Marois, 2004). The present study explores the neuronal substrates of this $1 \mathrm{~s}$ memory retention with an experimental paradigm that has been psychophysically well validated (Luck and Vogel, 1997; Todd and Marois, 2004; Vogel and Machizawa, 2004; Vogel et al., 2005). To grasp the temporal and spectral evolution of event-related ongoing activity, we first computed time-frequency (TF) representations (TFRs) of SL and oscillation amplitudes by using a bank of 36 Morlet wavelets from 3 to $90 \mathrm{~Hz}$. Similar to the analyses of fMRI data (Kriegeskorte et al., 2009), TF analyses of MEG data are susceptible to statistical inflation arising from circular data inspection and region of interest selection. To have a noncircular discovery strategy, we first pooled the statistical TF data across all brain regions by plotting for each TF element the fraction of statistically significant patches (or M/EEG sensors). To control for multiple comparisons, we removed the number of significant TF elements predicted to be false discoveries at the chosen alpha level, A. Because VWM is known to involve a widely distributed cortical network, this approach was expected to reveal those TF regions where large-scale cortical activity was modulated by VWM processing.

We first evaluated the changes from baseline level with Wilcoxon signed rank test for data averaged across the six memory loads. The TFR for SL showed that frequencies up to $40 \mathrm{~Hz}$ were phase locked to the stimulus onset for several hundreds of milliseconds from stimulus onset without a clear oscillatory component $(A=0.001$, FDR corrected) (Fig. $4 A$ ). The TFR of oscillation amplitudes revealed an amplitude increase in the theta/alpha band during the first $400 \mathrm{~ms}$ after stimulus onset, which coincided with the period of strong stimulus-locked activity $(A=0.01$, FDR corrected) (Fig. 4B). After this transient, the oscillation amplitudes were suppressed below the baseline level throughout the analyzed frequency range from theta/ alpha- to gamma-frequency bands. This suppression was also evident in broad-band filtered data (Fig. 4). We also confirmed that the recording method would not affect the observed pattern of SL or oscillation amplitudes by computing the TFRs separately for MEG gradiometers, magnetometers, and EEG electrodes. All three recording methods converged with source modeling on a similar result for both SL and amplitude dynamics. The fraction of significant sensors was greater than the fraction of significant source patches, suggesting that the source modeling was successful in disentangling cortical sources from their mixed sensor level signals (Fig. $4 A, B$; supplemental Fig. 6, available at www.jneurosci.org as supplemental material).

To investigate the impact of memory load on the oscillatory dynamics, we identified the brain regions in which SL or amplitude dynamics were correlated with memory load by using the Spearman's rank correlation test with the null hypothesis that the SL or amplitude values in the six individual memory load conditions were uncorrelated among the memory loads from one to six objects. Before testing, the individual subjects' data were rank transformed to ensure comparability across the population. The TFR of SL revealed an early positive correlation with memory load $(A=0.001$, FDR corrected) (Fig. $4 C$ ). The TFR of memory load-dependent amplitude changes showed an interesting pattern. Similar to SL and ERs, the amplitudes of theta/alpha- and highalpha-frequency bands were positively correlated with memory load during the first $200 \mathrm{~ms}$ after stimulus onset $(A=0.01$, FDR corrected) (Fig. $4 D$ ). The early positive effect thereafter changed into a negative correlation in the theta/alpha, high-alpha, and beta bands, which indicates that the amplitude suppression was even deeper at higher memory loads. The negative correlation, however, was sustained through the VWM retention period only 
in the theta/alpha band. The amplitudes of high-alpha and beta bands were positively correlated with memory load from $400 \mathrm{~ms}$ to the end of the VWM retention period, even though they were suppressed below the baseline level in the average condition.

On the basis of the TF mapping, we designed a set of broad-band FIR filters to capture the theta/alpha-, high-alpha-, beta-, gamma-, and high-gamma-frequency bands with a temporal resolution greater than that provided by the wavelets, and we also used a patch collection with a spatial resolution higher than was used in the wavelet-based analysis (see Materials and Methods). The amplitude time series obtained with these filters reproduced very well the phenomena discovered with wavelets ( $A=0.01$, FDR corrected) (Fig. $4 E)$, which shows that the results presented here are not qualitatively affected by the filtering or cortical parcellation approaches. However, the dynamics obtained with these broad-band filters revealed that also gamma- and high-gamma-band amplitudes were strengthened with increasing memory load.

\section{Cortical sources of retention period} modulations of oscillation amplitudes We used the FIR filtered theta/alpha-, high-alpha, beta-, gamma- and highgamma-band data to identify the cortical regions underlying the amplitude modulations during VWM retention. In data averaged across all six memory loads, the amplitudes were suppressed in distributed visual regions in striate, extrastriate, temporal, and parietal cortices $(A=0.01)$ (Fig. $5 A$ ). In the beta and gamma bands, the suppression was most pronounced in medial occipital and parietal cortices, but also observed in occipitotemporal and temporal visual areas. In addition, the suppression was observed in the same cortical regions during the early $(400-700 \mathrm{~ms}$ from sample stimulus onset) and late (700-1000 ms from sample stimulus onset) parts of the retention period (supplemental Fig. 7, available at www.jneurosci. org as supplemental material).

Increasing memory load was associated with a further suppression of amplitudes in the theta/alpha- and high-alpha bands in the occipital, occipitotemporal, and parietal regions (Fig. 5B). The amplitudes in the high-alpha, beta, gamma, and high-gamma bands, and very weakly in the theta/alpha band, were strengthened in widespread frontoparietal cortical regions, including the LPFC, precentral and postcentral regions, and cingulate, insular, and orbito-

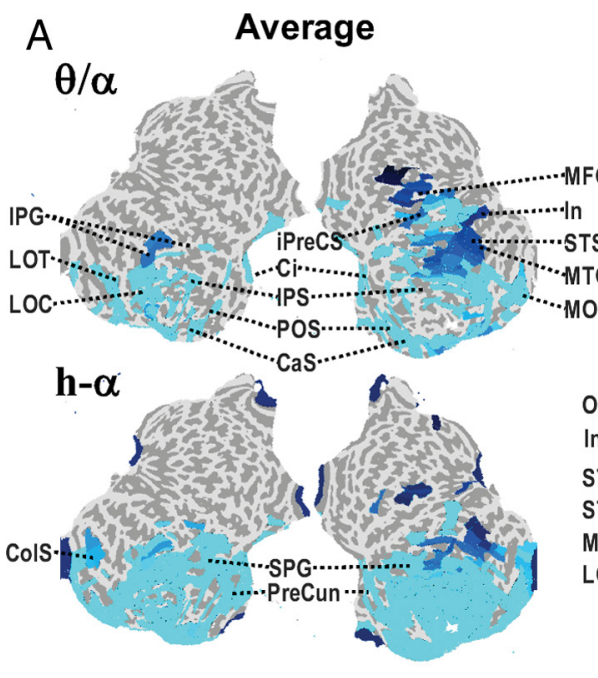

B
Load

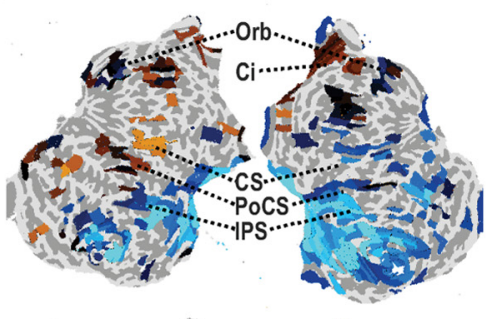

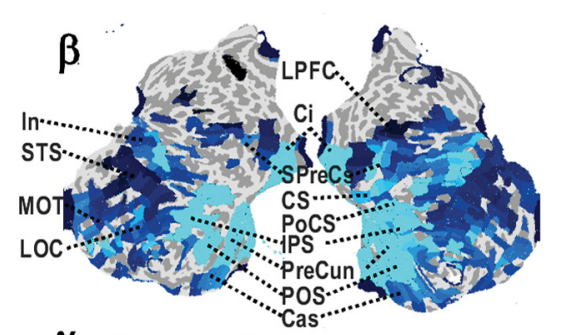
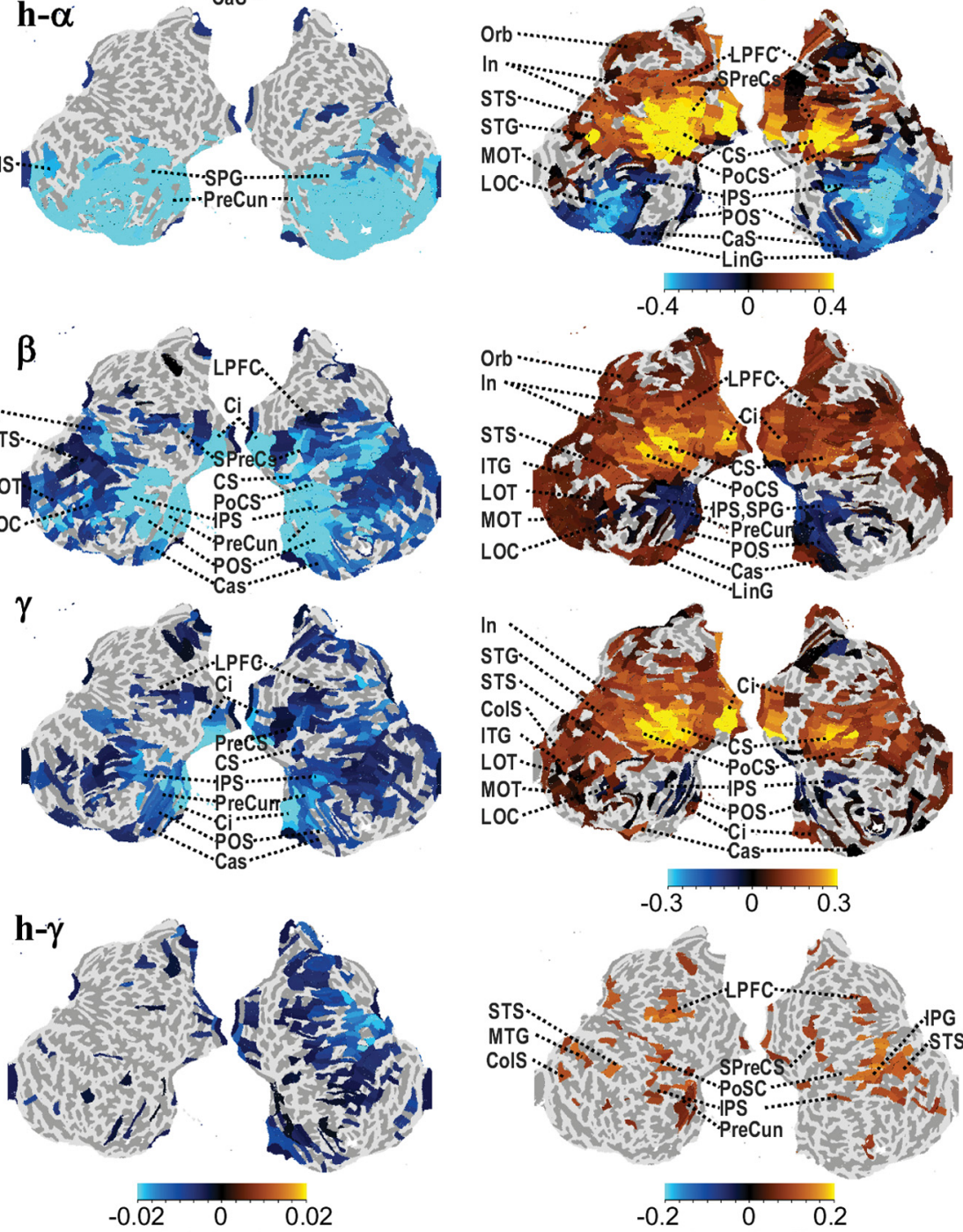

Rel. Ampl.

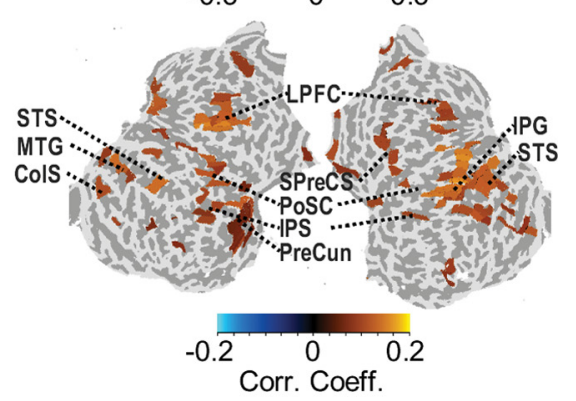

Figure 5. Oscillation amplitudes were suppressed during the memory retention period in several visual and central cortical regions but were nevertheless positively correlated with memory load in a large-scale cortical network. $\boldsymbol{A}$, Localization of amplitude modulation in the average condition during the retention period from 0.4 to 1 s from sample stimulus onset in different frequency bands. The negative amplitude modulation is observed throughout the visual regions to the posterior parts of the lateral frontal cortex. The color values illustrate the mean relative oscillation amplitude change from the baseline level in those cortical regions that were significantly modulated in this time window ( $p<0.001$, corrected). See supplemental Figure 7 (available at www.jneurosci.org as supplemental material) for these data in time windows from 0.4 to 0.7 and 0.7 to 1 s. $\boldsymbol{B}$, Correlation of cortical oscillation amplitudes with memory load during the retention period. The color values indicate the mean Spearman's correlation coefficient in those cortical regions that were significant positively or negatively correlated with memory load ( $p<0.01$, corrected). The amplitudes of high-alpha (h- $\alpha)-$, beta $(\beta)-$, gamma $(\gamma)-$, and high-gamma $(h-\gamma)$-frequency bands were strengthened in frontoparietal regions as well as in insula and cingulate. In visual cortical regions in the occipital and occipitotemporal cortices, the $h$ - $\alpha$-band amplitudes were negatively correlated with memory load, but the $\beta$ - and $\gamma$-band amplitudes were positively correlated. Abbreviations are as in Figure 2. MFG, Middle frontal gyrus; PreCun, precuneus; PoCS, postcentral sulcus. 
A

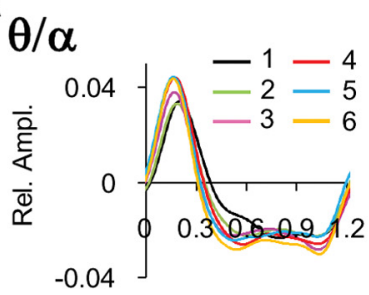

h- $\alpha$

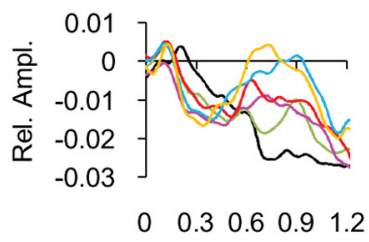

$\beta$

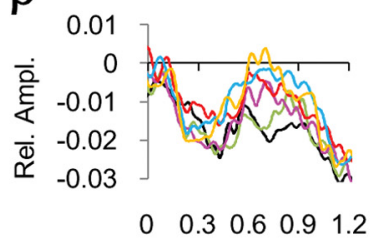

$\gamma$

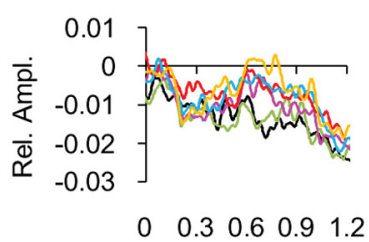

h- $\gamma$

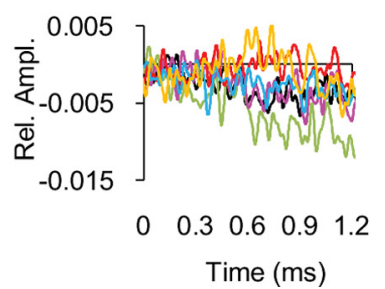

B
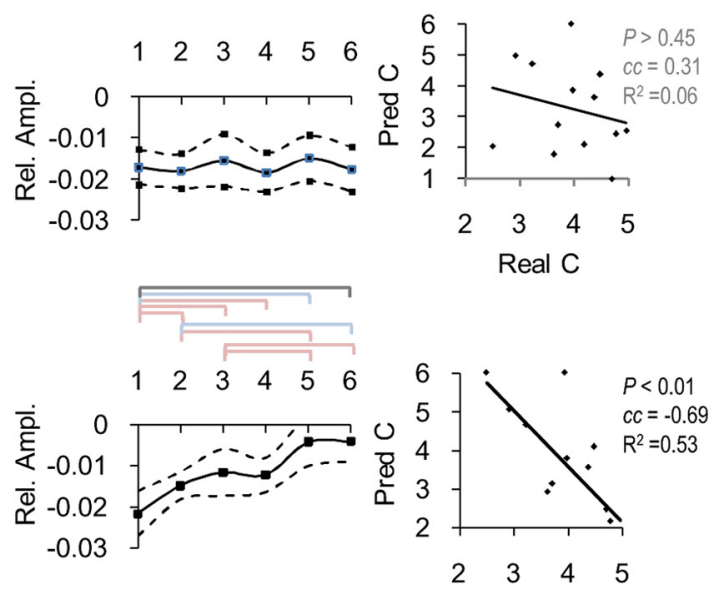

Real C
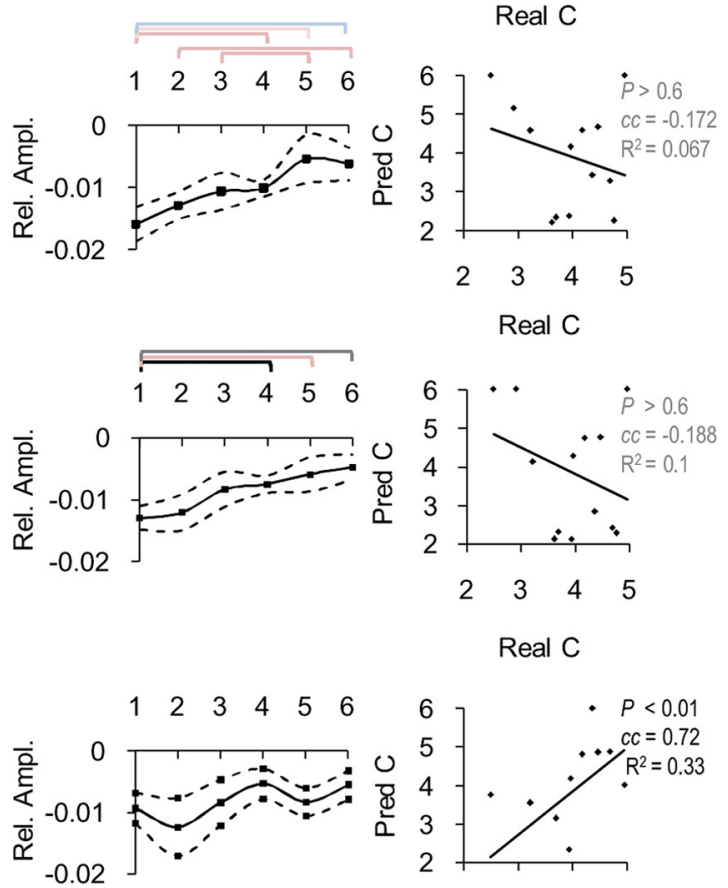

VWM load

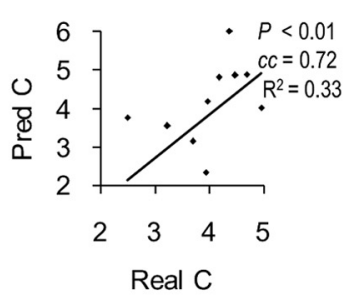

Figure 6. Waveform visualizations and post hoc statistics for the memory load-dependent retention period oscillations. $\boldsymbol{A}$, Broad-band oscillation amplitudes averaged across those cortical regions that were significantly correlated with memory load (Fig. 5). $\boldsymbol{B}$, The oscillation amplitudes were averaged across the late retention period and within each subject $(700-1000 \mathrm{~ms}$, black line, dashed lines indicate the SEM across subjects). The brackets indicate the memory load pairs that were significantly different in post hoc paired $t$ tests (black lines: $p<0.01$, corrected with $N_{\text {tests }}=15$; gray lines: $p<0.05$, corrected; blue lines: $p<$ 0.01 , uncorrected; and pink lines: $p<0.05$, uncorrected). These data corroborate the findings that $h-\alpha-, \beta$-, and $\gamma$-band oscillation amplitudes are positively correlated with memory load even though they remain suppressed below the baseline level. C, The correlation between the individual behavioral memory capacity values (Real C) with the capacity values predicted by the dependence of the oscillation amplitudes on memory load (Pred. C). Rel. Ampl., Relative amplitude. Other abbreviations are as in Figure 4.

frontal cortices. Interestingly, the beta- and gamma-band amplitudes were, in addition, strengthened in several visual regions in the occipital and occipitotemporal, and temporal cortices that are known to play a central role in the formation of visual object representations (Riesenhuber and Poggio, 2002; Grill-Spector and Malach, 2004; Konen and Kastner, 2008). While the anterior positive correlations remained very similar at $A=0.001$, the posterior positive correlations in beta and gamma bands did not exceed this higher alpha level. High-gamma-band amplitudes were positively correlated with memory load in LPFC, posterior parietal, temporal, and insular cortical regions. Importantly, the network of load-dependent highgamma oscillations was spatially stable throughout the retention period (see supplemental Fig. 7, available at www.jneurosci. org as supplemental material).

The Morlet wavelet analysis approach revealed essentially the same frontoparietal pattern, indicating that the parcellation scheme did not affect the primary results ( $A=0.01$, FDR corrected) (supplemental Fig. 8, available at www.jneurosci.org as supplemental material). However, the memory load-dependent strengthening of beta- and gamma-band amplitudes in visual regions did not exceed the significance threshold, which is likely to be caused by a greater spectral variability in these bands across the subjects.

\section{Correlation of oscillation amplitudes with memory load and behavioral capacity}

The results have so far clearly indicated that the amplitudes of high-alpha-, beta-, gamma-, and high-gamma-band oscillations were positively correlated with memory load in a frontoparietal network (Fig. 5B). To further characterize this phenomenon with post hoc analyses, we estimated the amplitudes in all frequency bands separately for each memory load and individual subject as an average across the cortical regions that were positively correlated with memory load (Fig. 5B). These data corroborated that the amplitudes were indeed memory load dependent during the memory retention period (Fig. 6A). Evaluation of mean amplitudes averaged over the latter half of the retention period (700-1000 ms) showed that oscillation amplitudes were systematically increased with memory load, but with a biphasic shape and a plateau at three to four objects (Fig. 6B). The analysis for the Morlet wavelet-filtered data showed similarly that both the strengths and fraction of significant cortical regions were systematically modulated by the increasing memory load (supplemental Fig. $9 A-D$, available at www. jneurosci.org as supplemental material).

To address the link between the retention period amplitude modulations and individual behavioral VWM capacity, we used the same approach as above for ERs (Fig. 3 ). In the bilateral frontoparietal regions, the plateau onsets of the high-alpha-band amplitudes were negatively correlated with memory capacity (Fig. 6C), implying that in high-capacity subjects, the load-dependent alpha strengthening leveled off at lower memory loads than in low-capacity subjects. On the other hand, despite a similar negative trend, neither beta- nor gamma-band 
amplitudes were significantly correlated with the memory capacity. Interestingly, although the high-gamma-band amplitudes were only weakly correlated with the VWM load, the plateau onsets in highgamma band were clearly positively correlated with the individual VWM capacity.

We also investigated the correlation of the load-dependent amplitude suppression with memory capacity. We first estimated the relative amplitudes from those cortical regions that were negatively correlated with the memory load (supplemental Fig. 10A, available at www.jneurosci.org as supplemental material). The amplitudes were then averaged over the retention period (supplemental Fig. $10 \mathrm{~B}$, available at www.jneurosci. org as supplemental material). Last, we estimated the correlation between the predicted and real capacity values (supplemental Fig. 10C, available at www.jneurosci.org as supplemental material). The amplitude suppression was not, however, significantly correlated with the individual memory capacity in any of the studied frequency bands.

Finally, to corroborate the group analysis approach, we asked whether low- and high-capacity subject populations were associated with qualitatively and phenomenologically similar retention period oscillation dynamics. The subject group was divided into two halves by individual memory capacity, and cortical regions where oscillation amplitudes were correlated memory load were identified with Spearman's rank correlation test $(p<0.01$, FDR corrected) as above (Fig. 7). The left-lateralized but bilaterally highly significant frontoparietal highalpha-, beta-, and gamma-band oscillations were observed in both subject populations, although they were more pronounced for the high-capacity subjects. On the other hand, only the high-capacity group was associated with a significant memory load correlation in the high-gamma band. The apparent absence of memory load-dependent high gamma in the low-capacity group may, however, be associated with the overall poor signal-to-noise ratio in this frequency band. Interestingly, the amplitude suppression in the high-alpha band was less pronounced for the high- than the low-capacity subjects.

\section{Discussion}

Stimulus processing and VWM encoding during early stimulus-locked activity The processing of sample stimuli was associated with a rapid spread of activity during the first $\sim 200 \mathrm{~ms}$ from occipital to temporal and parietal cortices. These regions are known to underlie the formation of visual object representations (Singer, 1999; Riesenhuber and Poggio, 2002; Grill-Spector and Malach, 2004; Konen and Kastner, 2008). The P1 component ( $\sim 120 \mathrm{~ms})$ was

\section{A Load, Low Capacity}
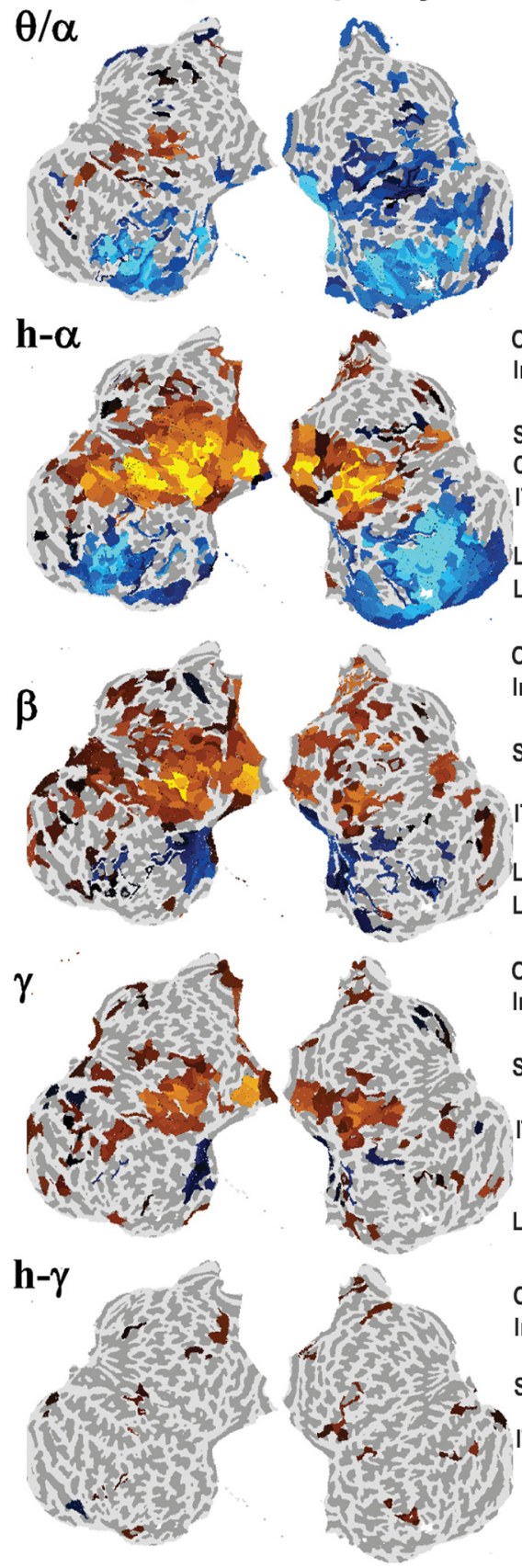

Figure 7. Low-and high-capacity subject groups were associated with qualitatively and phenomenologically similar retention period oscillation dynamics. The color values indicate the mean Spearman's correlation coefficient in the time window from 0.4 to $1 \mathrm{~s}$ in cortical regions where the oscillation amplitudes significantly positively or negatively correlated with memory load (see Fig. 5 for details; $p<0.01$, corrected). Abbreviations are as in Figures 2 and 4. CS, Central sulcus.

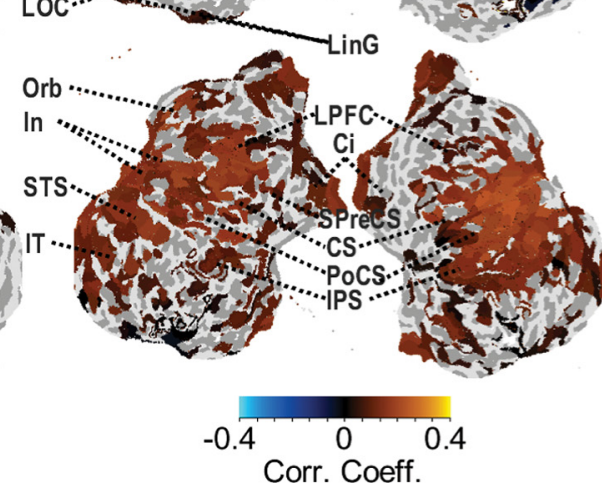

\section{B Load, High Capacity}
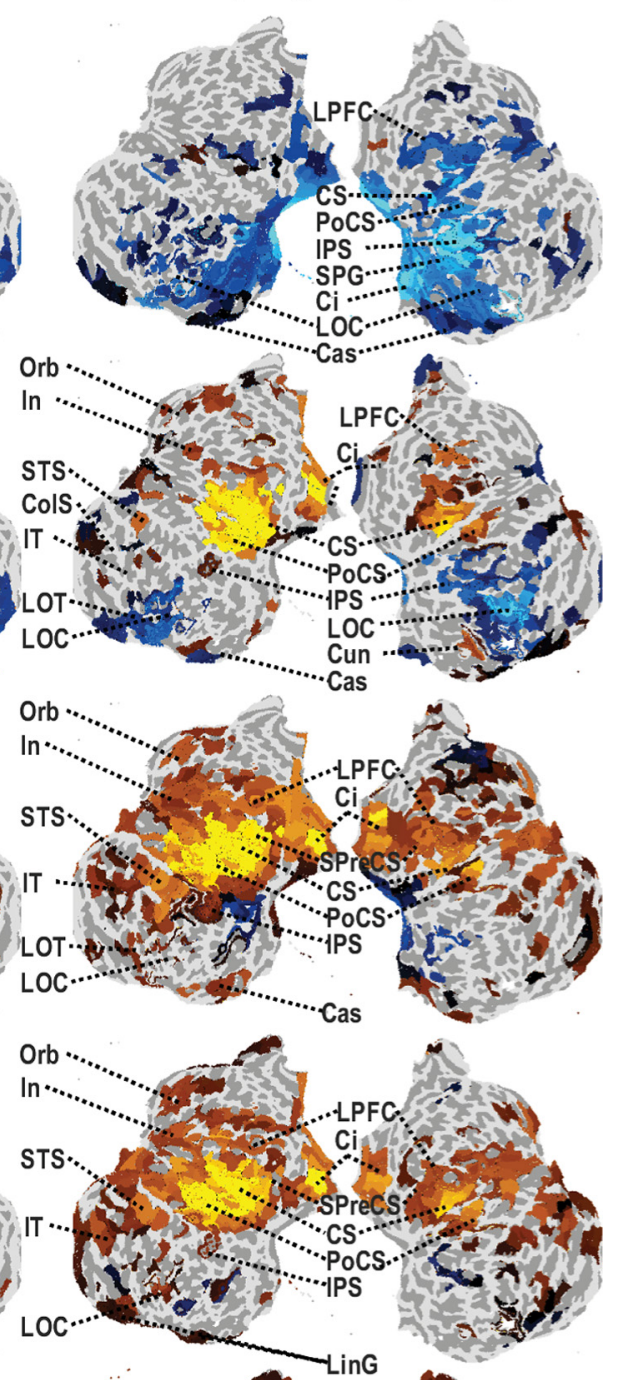

Corr. Coeff.

linearly strengthened by the VWM load, whereas the magnitude of the N1 component ( $\sim 190 \mathrm{~ms}$ ) plateaued with increasing memory load. This plateau onset was positively correlated with individual behavioral VWM capacity in the early visual regions. Interestingly, during the $\mathrm{N} 1$ component, activity in visual regions was accompanied by concurrent activation of frontal and parietal structures. It is thus likely that the stage of stimulus processing and memory encoding already reflected in the N1 component, 
frontoparieto-sensory interaction, and frontoparietal topdown modulation of early visual regions give rise to individual behavioral memory capacity limits.

\section{Localization of oscillation amplitude dynamics during VWM retention}

Evoked stimulus processing was followed by amplitude suppression in posterior, central, and frontal VWM-related cortical areas in frequency bands from 5 to $90 \mathrm{~Hz}$. A similar wide-band amplitude suppression has been observed to follow sensory stimuli in MEG (Palva et al., 2005) and to characterize the VWM retention period in human EEG (Medendorp et al., 2007) and in direct cortical (Axmacher et al., 2007; Meltzer et al., 2008) recordings. In the present and prior VWM MEG/EEG data, increasing memory load strengthened the amplitudes of high-alpha- (Busch and Herrmann, 2003; Leiberg et al., 2006; Grimault et al., 2009; Haenschel et al., 2009), beta- (Leiberg et al., 2006), and gamma(Tallon-Baudry et al., 1998; Osipova et al., 2006; Haenschel et al., 2009) frequency bands. In the present data, the amplitudes of high-alpha, beta-, gamma-, and high-gamma-frequency bands were positively correlated with memory load in several frontal, parietal, and temporal regions as well as in the cingulate and insular cortices. Prior MEG studies suggest that the positive memory load dependence in the $\alpha$ band originates from occipitoparietal brain regions (Jensen et al., 2002; Osipova et al., 2006; Medendorp et al., 2007), which is in contrast with the frontal sources found here. However, the central difference in these studies is the time window of the effect. In the present study, the retention period effects are observed in a time window of 0.5 to $1 \mathrm{~s}$, and in those cited above, in a time window from 1 to $3 \mathrm{~s}$. In fact, these findings together constitute neurophysiological support for the notion that there is transition in VWM maintenance mechanisms at $\sim 1$ s (Todd and Marois, 2004).

These data thus show load-dependently strengthened oscillation amplitudes in several cortical regions, which have earlier been identified in VWM studies using fMRI (Prabhakaran et al., 2000; Rowe et al., 2000; Munk et al., 2002; Pessoa et al., 2002; Linden et al., 2003; Todd and Marois, 2004). Although these frontal and parietal regions are well recognized cortical substrates for WM function, a number of other areas also have been revealed by fMRI and here with M/EEG. While the functional roles of cingulate, orbital, and insular cortices in VWM have remained unclear, it is intriguing that their multiband amplitude dynamics in the present study were similar to those observed in frontoparietal regions. The cingulate, orbital, and insular structures were also strongly phase synchronized with the frontoparietal networks in these frequency bands in our prior work (Palva et al., $2010 \mathrm{~b})$. These results together suggest that in addition to classical VWM regions, these regions also contribute to the VWM maintenance in subsecond time scales.

\section{The functional role of neuronal oscillations in VWM retention}

Three aspects of the present data yield insight into the functional roles of oscillatory activity in VWM: memory load dependence, anatomical localization, and correlation with behavior. Frontal and parietal regions are thought to underlie attentional and central executive functions in VWM as indexed by fMRI (Prabhakaran et al., 2000; Rowe et al., 2000; Sakai et al., 2002; Curtis and D'Esposito, 2003; Petrides, 2005) and lesion (Voytek and Knight, 2010) studies. Hence, the frontoparietal localization of the positive memory load dependence of high-alpha, beta, gamma, and high-gamma oscillations implies that they are associated with attentional functions of VWM retention. This finding thus supports earlier suggestions for a role for high-alpha (Klimesch et al., 2007; Palva and Palva, 2007), beta (Gross et al., 2004; Buschman and Miller, 2007), gamma, and high-gamma (Fries, 2009) oscillations in attention. Beta- and gamma-frequency band oscillations were also strengthened over several visual cortical regions, including the inferotemporal and occipitotemporal regions that are associated with visual object processing (Riesenhuber and Poggio, 2002; GrillSpector and Malach, 2004; Konen and Kastner, 2008), which is in line with the idea that beta- and gamma-band oscillations support the maintenance of object representations in VWM (TallonBaudry et al., 1998; Jokisch and Jensen, 2007; Palva et al., 2010b). Thus, in terms of activity localized to sensory brain regions, the present data are in line with the currently prevailing idea of distinct functional roles for alpha vs beta-gamma oscillations. Alpha oscillation amplitude dynamics are often interpreted along the lines of the inhibition hypothesis (Pfürtscheller, 2003; Klimesch et al., 2007), which posits that large alpha amplitudes in a given region reflect the disengagement of that region from taskrelevant processing and that alpha-band amplitude suppression reflects a release from inhibition of that region. However, both the overall amplitude suppression and the positive memory load dependence in high-alpha, beta, gamma, and high-gamma bands were largely colocalized in the frontoparietal network, which is difficult to reconcile with the inhibition hypothesis. It is thus possible that the phenomenology and functional roles of alpha and perhaps of also other oscillations depend on the level of cortical hierarchy (Bollimunta et al., 2008).

\section{Neuronal correlates of behavioral VWM capacity}

A number of fMRI studies report that activity in IPS and LOC may predict the behavioral VWM capacity (Todd and Marois, 2004; Xu and Chun, 2006; Robitaille et al., 2010). In MEG and EEG, the lateralization of ER components (Vogel and Machizawa, 2004; Robitaille et al., 2010), posterior alpha-band amplitude modulations (Sauseng et al., 2009), and the strength of interareal phase synchrony in alpha and beta bands (Palva et al., 2010b) are also correlated with the individual VWM capacity. In the present study, we found that the plateau in the load-dependent magnitude of the $\mathrm{N} 1$ evoked response component in early visual regions was positively correlated with individual capacity. During VWM retention period, the memory load-dependent strengthening of high-alpha-band amplitudes in the frontoparietal regions was negatively correlated with the individual capacity showing that high-alpha amplitude peaked or plateaued at progressively lower memory loads for higher capacity subjects. This suggests that enhanced high-alpha oscillations are associated with attentional control when the number of to-be-memorized items exceeds the individual memory capacity. In contrast, the plateau in the loaddependent strengthening of high-gamma oscillations was positively correlated with individual VWM capacity, which supports the concept that high-gamma oscillations underlie integrated neuronal object representations (Singer, 1999) that are sustained in an active state during VWM maintenance. Accordingly, the memory load dependence of high-gamma oscillations was also more pronounced in high- than in low-capacity subjects. However, the high- and low-capacity subject groups were overall characterized by qualitatively similar patterns of amplitude modulations, which indicates that these data do not warrant strongly dichotomous interpretations on subject classification. Overall, a growing body of evidence demonstrates that the neuronal mechanisms underlying the limited human VWM capacity may be more heterogeneous and complex 
than previously conceived and that these mechanisms include both encoding- and retention-related neuronal activities.

\section{The relationship between the oscillation amplitudes and interareal synchrony}

We have recently shown that interareal 1:1 phase synchrony in alpha, beta, and gamma bands is load-dependently strengthened in a frontoparietal network (Palva et al., 2010b) wherein also the oscillation amplitudes were load-dependently strengthened. This raised the question of whether the synchronization estimates were biased by an improved signal-to-noise ratio associated with the strengthening of amplitudes. The concurrent changes in phase synchrony were, however, much stronger than those predicted by SNR changes (Palva et al., 2010b), suggesting that the positive correlation of frontoparietal oscillation amplitudes with memory load could be a reflection of the colocalized strengthening of short-range phase synchronization. In line with this notion, the network topologies in the high-alpha and beta bands were more clustered and small-world-like than those in theta- or gamma-frequency bands (Palva et al., 2010a). Hence, phase synchrony in dense core-like structures is likely to be positively correlated with local amplitude changes, while in the topologically less clustered brain regions phase synchrony and local amplitude dynamics can be more independent. In line with previous studies (Freunberger et al., 2008; Doesburg et al., 2009; Freunberger et al., 2009), interareal synchronization was enhanced above baseline levels throughout the memory retention period (Palva et al., 2010b), whereas the oscillation amplitudes were suppressed below the baseline. This important phenomenological dichotomy between oscillation amplitudes and synchrony shows that local amplitude modulations and long-range interareal phase synchronization are dissociable phenomena that conceivably reflect local processing vs large-scale interactions.

\section{References}

Axmacher N, Mormann F, Fernández G, Cohen MX, Elger CE, Fell J (2007) Sustained neural activity patterns during working memory in the human medial temporal lobe. J Neurosci 27:7807-7816.

Baddeley A (1996) The fractionation of working memory. Proc Natl Acad Sci U S A 93:13468-13472.

Bollimunta A, Chen Y, Schroeder CE, Ding M (2008) Neuronal mechanisms of cortical alpha oscillations in awake-behaving macaques. J Neurosci 28:9976-9988.

Busch NA, Herrmann CS (2003) Object-load and feature-load modulate EEG in a short-term memory task. Neuroreport 14:1721-1724.

Buschman TJ, Miller EK (2007) Top-down versus bottom-up control of attention in the prefrontal and posterior parietal cortices. Science 315:1860-1862.

Curtis CE, D'Esposito M (2003) Persistent activity in the prefrontal cortex during working memory. Trends Cogn Sci 7:415-423.

Dale AM, Fischl B, Sereno MI (1999) Cortical surface-based analysis. I. Segmentation and surface reconstruction. Neuroimage 9:179-194.

de Munck JC, Bijma F (2010) How are evoked responses generated? The need for a unified mathematical framework. Clin Neurophysiol 121:127-129.

Desikan RS, Ségonne F, Fischl B, Quinn BT, Dickerson BC, Blacker D, Buckner RL, Dale AM, Maguire RP, Hyman BT, Albert MS, Killiany RJ (2006) An automated labeling system for subdividing the human cerebral cortex on MRI scans into gyral based regions of interest. Neuroimage 31:968-980.

Doesburg SM, Green JJ, McDonald JJ, Ward LM (2009) From local inhibition to long-range integration: a functional dissociation of alpha-band synchronization across cortical scales in visuospatial attention. Brain Res 1303:97-110.

Fischl B, Sereno MI, Dale AM (1999) Cortical surface-based analysis. II: inflation, flattening, and a surface-based coordinate system. Neuroimage 9:195-207.
Fischl B, Liu A, Dale AM (2001) Automated manifold surgery: constructing geometrically accurate and topologically correct models of the human cerebral cortex. IEEE Trans Med Imaging 20:70-80.

Fischl B, Salat DH, Busa E, Albert M, Dieterich M, Haselgrove C, van der Kouwe A, Killiany R, Kennedy D, Klaveness S, Montillo A, Makris N, Rosen B, Dale AM (2002) Whole brain segmentation: automated labeling of neuroanatomical structures in the human brain. Neuron 33:341-355.

Fischl B, van der Kouwe A, Destrieux C, Halgren E, Ségonne F, Salat DH, Busa E, Seidman LJ, Goldstein J, Kennedy D, Caviness V, Makris N, Rosen B, Dale AM (2004) Automatically parcellating the human cerebral cortex. Cereb Cortex 14:11-22.

Freunberger R, Klimesch W, Griesmayr B, Sauseng P, Gruber W (2008) Alpha phase coupling reflects object recognition. Neuroimage 42:928-935.

Freunberger R, Fellinger R, Sauseng P, Gruber W, Klimesch W (2009) Dissociation between phase-locked and non-phaselocked alpha oscillations in a working memory task. Hum Brain Mapp 30:3417-3425.

Fries P (2009) Neuronal gamma-band synchronization as a fundamental process in cortical computation. Annu Rev Neurosci 32:209-224.

Grill-Spector K, Malach R (2004) The human visual cortex. Annu Rev Neurosci 27:649-677.

Grimault S, Robitaille N, Grova C, Lina JM, Dubarry AS, Jolicoeur P (2009) Oscillatory activity in parietal and dorsolateral prefrontal cortex during retention in visual short-term memory: additive effects of spatial attention and memory load. Hum Brain Mapp 30:3378-3392.

Gross J, Schmitz F, Schnitzler I, Kessler K, Shapiro K, Hommel B, Schnitzler A (2004) Modulation of long-range neural synchrony reflects temporal limitations of visual attention in humans. Proc Natl Acad Sci U S A 101:13050-13055.

Haegens S, Osipova D, Oostenveld R, Jensen O (2010) Somatosensory working memory performance in humans depends on both engagement and disengagement of regions in a distributed network. Hum Brain Mapp 31:26-35.

Haenschel C, Bittner RA, Waltz J, Haertling F, Wibral M, Singer W, Linden DE, Rodriguez E (2009) Cortical oscillatory activity is critical for working memory as revealed by deficits in early-onset schizophrenia. J Neurosci 29:9481-9489.

Hämäläinen MS, Ilmoniemi RJ (1994) Interpreting magnetic fields of the brain: minimum norm estimates. Med Biol Eng Comput 32:35-42.

Hämäläinen MS, Sarvas J (1989) Realistic conductivity geometry model of the human head for interpretation of neuromagnetic data. IEEE Trans Biomed Eng 36:165-171.

Hauk O, Wakeman DG, Henson R (2011) Comparison of noise-normalized minimum norm estimates for MEG analysis using multiple resolution metrics. Neuroimage 54:1966-1974.

Howard MW, Rizzuto DS, Caplan JB, Madsen JR, Lisman J, AschenbrennerScheibe R, Schulze-Bonhage A, Kahana MJ (2003) Gamma oscillations correlate with working memory load in humans. Cereb Cortex 13:1369-1374.

Jensen O, Gelfand J, Kounios J, Lisman JE (2002) Oscillations in the alpha band $(9-12 \mathrm{hz})$ increase with memory load during retention in a shortterm memory task. Cereb Cortex 12:877-882.

Jokisch D, Jensen O (2007) Modulation of gamma and alpha activity during a working memory task engaging the dorsal or ventral stream. J Neurosci 27:3244-3251

Kaiser J, Heidegger T, Wibral M, Altmann CF, Lutzenberger W (2008) Distinct gamma-band components reflect the short-term memory maintenance of different sound lateralization angles. Cereb Cortex 18:2286-2295.

Klimesch W, Sauseng P, Hanslmayr S (2007) EEG alpha oscillations: the inhibition-timing hypothesis. Brain Res Rev 53:63-88.

Konen CS, Kastner S (2008) Two hierarchically organized neural systems for object information in human visual cortex. Nat Neurosci 11:224-231.

Kriegeskorte N, Simmons WK, Bellgowan PS, Baker CI (2009) Circular analysis in systems neuroscience: the dangers of double dipping. Nat Neurosci 12:535-540.

Leiberg S, Lutzenberger W, Kaiser J (2006) Effects of memory load on cortical oscillatory activity during auditory pattern working memory. Brain Res 1120:131-140.

Lin FH, Belliveau JW, Dale AM, Hämäläinen MS (2006) Distributed current estimates using cortical orientation constraints. Hum Brain Mapp 27:1-13.

Linden DE, Bittner RA, Muckli L, Waltz JA, Kriegeskorte N, Goebel R, Singer 
W, Munk MH (2003) Cortical capacity constraints for visual working memory: dissociation of fMRI load effects in a fronto-parietal network. Neuroimage 20:1518-1530.

Luck SJ, Vogel EK (1997) The capacity of visual working memory for features and conjunctions. Nature 390:279-281.

Mainy N, Kahane P, Minotti L, Hoffmann D, Bertrand O, Lachaux JP (2007) Neural correlates of consolidation in working memory. Hum Brain Mapp 28:183-193.

Makeig S, Westerfield M, Jung TP, Enghoff S, Townsend J, Courchesne E, Sejnowski TJ (2002) Dynamic brain sources of visual evoked responses. Science 295:690-694.

Medendorp WP, Kramer GF, Jensen O, Oostenveld R, Schoffelen JM, Fries P (2007) Oscillatory activity in human parietal and occipital cortex shows hemispheric lateralization and memory effects in a delayed double-step saccade task. Cereb Cortex 17:2364-2374.

Meltzer JA, Zaveri HP, Goncharova II, Distasio MM, Papademetris X, Spencer SS, Spencer DD, Constable RT (2008) Effects of working memory load on oscillatory power in human intracranial EEG. Cereb Cortex 18:1843-1855.

Mohr HM, Goebel R, Linden DE (2006) Content- and task-specific dissociations of frontal activity during maintenance and manipulation in visual working memory. J Neurosci 26:4465-4471.

Monto S, Palva S, Voipio J, Palva JM (2008) Very slow EEG fluctuations predict the dynamics of stimulus detection and oscillation amplitudes in humans. J Neurosci 28:8268-8272.

Morgan HM, Jackson MC, Klein C, Mohr H, Shapiro KL, Linden DE (2010) Neural signatures of stimulus features in visual working memory-a spatiotemporal approach. Cereb Cortex 20:187-197.

Mosher JC, Leahy RM, Lewis PS (1999) EEG and MEG: forward solutions for inverse methods. IEEE Trans Biomed Eng 46:245-259.

Munk MH, Linden DE, Muckli L, Lanfermann H, Zanella FE, Singer W, Goebel R (2002) Distributed cortical systems in visual short-term memory revealed by event-related functional magnetic resonance imaging. Cereb Cortex 12:866-876.

Nenonen JT, Hämäläinen MS, Ilmoniemi RJ (1994) Minimum-norm estimation in a boundary-element torso model. Med Biol Eng Comput 32:43-48.

Nikulin VV, Linkenkaer-Hansen K, Nolte G, Lemm S, Müller KR, Ilmoniemi RJ, Curio G (2007) A novel mechanism for evoked responses in the human brain. Eur J Neurosci 25:3146-3154.

Onton J, Delorme A, Makeig S (2005) Frontal midline EEG dynamics during working memory. Neuroimage 27:341-356.

Osipova D, Takashima A, Oostenveld R, Fernández G, Maris E, Jensen O (2006) Theta and gamma oscillations predict encoding and retrieval of declarative memory. J Neurosci 26:7523-7531.

Palva S, Palva JM (2007) New vistas for alpha-frequency band oscillations. Trends Neurosci 30:150-158.

Palva S, Linkenkaer-Hansen K, Näätänen R, Palva JM (2005) Early neural correlates of conscious somatosensory perception. J Neurosci 25: $5248-5258$

Palva S, Monto S, Palva JM (2010a) Graph properties of synchronized cortical networks during visual working memory maintenance. Neuroimage 49:3257-3268.

Palva JM, Monto S, Kulashekhar S, Palva S (2010b) Neuronal synchrony reveals working memory networks and predicts individual memory capacity. Proc Natl Acad Sci U S A 107:7580-7585.

Pessoa L, Gutierrez E, Bandettini P, Ungerleider L (2002) Neural correlates of visual working memory: FMRI amplitude predicts task performance. Neuron 35:975-987.

Petrides M (2005) Lateral prefrontal cortex: architectonic and functional organization. Philos Trans R Soc Lond B Biol Sci 360:781-795.

Pfürtscheller G (2003) Induced oscillations in the alpha band: functional meaning. Epilepsia 44 [Suppl 12]:2-8.
Prabhakaran V, Narayanan K, Zhao Z, Gabrieli JD (2000) Integration of diverse information in working memory within the frontal lobe. Nat Neurosci 3:85-90.

Raghavachari S, Kahana MJ, Rizzuto DS, Caplan JB, Kirschen MP, Bourgeois B, Madsen JR, Lisman JE (2001) Gating of human theta oscillations by a working memory task. J Neurosci 21:3175-3183.

Riesenhuber M, Poggio T (2002) Neural mechanisms of object recognition. Curr Opin Neurobiol 12:162-168

Robitaille N, Marois R, Todd J, Grimault S, Cheyne D, Jolicoeur P (2010) Distinguishing between lateralized and nonlateralized brain activity associated with visual short-term memory: FMRI, MEG, and EEG evidence from the same observers. Neuroimage 53:1334-1345.

Rowe JB, Toni I, Josephs O, Frackowiak RS, Passingham RE (2000) The prefrontal cortex: response selection or maintenance within working memory? Science 288:1656-1660.

Sakai K, Rowe JB, Passingham RE (2002) Active maintenance in prefrontal area 46 creates distractor-resistant memory. Nat Neurosci 5:479-484.

Sauseng P, Klimesch W, Heise KF, Gruber WR, Holz E, Karim AA, Glennon M, Gerloff C, Birbaumer N, Hummel FC (2009) Brain oscillatory substrates of visual short-term memory capacity. Curr Biol 19:1846-1852.

Scheeringa R, Petersson KM, Oostenveld R, Norris DG, Hagoort P, Bastiaansen MC (2009) Trial-by-trial coupling between EEG and BOLD identifies networks related to alpha and theta EEG power increases during working memory maintenance. Neuroimage 44:1224-1238.

Schroeder CE, Lakatos P (2009) Low-frequency neuronal oscillations as instruments of sensory selection. Trends Neurosci 32:9-18.

Sederberg PB, Schulze-Bonhage A, Madsen JR, Bromfield EB, McCarthy DC, Brandt A, Tully MS, Kahana MJ (2007) Hippocampal and neocortical gamma oscillations predict memory formation in humans. Cereb Cortex 17:1190-1196.

Ségonne F, Dale AM, Busa E, Glessner M, Salat D, Hahn HK, Fischl B (2004) A hybrid approach to the skull stripping problem in MRI. Neuroimage 22:1060-1075.

Singer W (1999) Neuronal synchrony: a versatile code for the definition of relations? Neuron 24:49-65, 111-125.

Singer W (2009) Distributed processing and temporal codes in neuronal networks. Cogn Neurodyn 3:189-196.

Sinkkonen J, Tiitinen H, Näätänen R (1995) Gabor filters: an informative way for analysing event-related brain activity. J Neurosci Methods 56:99-104

Tallon-Baudry C, Bertrand O, Delpuech C, Pernier J (1996) Stimulus specificity of phase-locked and non-phase-locked $40 \mathrm{~Hz}$ visual responses in human. J Neurosci 16:4240-4249.

Tallon-Baudry C, Bertrand O, Peronnet F, Pernier J (1998) Induced gamma-band activity during the delay of a visual short-term memory task in humans. J Neurosci 18:4244-4254

Todd JJ, Marois R (2004) Capacity limit of visual short-term memory in human posterior parietal cortex. Nature 428:751-754.

Vogel EK, Machizawa MG (2004) Neural activity predicts individual differences in visual working memory capacity. Nature 428:748-751.

Vogel EK, McCollough AW, Machizawa MG (2005) Neural measures reveal individual differences in controlling access to working memory. Nature 438:500-503.

Voytek B, Knight RT (2010) Prefrontal cortex and basal ganglia contributions to visual working memory. Proc Natl Acad Sci U S A 107: 18167-18172.

Wibral M, Turi G, Linden DE, Kaiser J, Bledowski C (2008) Decomposition of working memory-related scalp ERPs: crossvalidation of fMRIconstrained source analysis and ICA. Int J Psychophysiol 67:200-211.

Xu Y, Chun MM (2006) Dissociable neural mechanisms supporting visual short-term memory for objects. Nature 440:91-95.

Zhang W, Luck SJ (2008) Discrete fixed-resolution representations in visual working memory. Nature 453:233-235. 